\title{
Manual RC Bridge Analysis and Designing
}

\author{
Applied Science
}

Ayaanle Maxamed Ali

Department: Civil Engineering

Program: Culvert Project 


\begin{abstract}
Bridges is a structure intended to carry the road traffic or other moving load to pass through the obstacles. The bridge may be roadway bridge, railway bridge,a canal, pipelines, etc. the main obstacles can be river, valley, and others.Bridges are very important in the modern roads and railway transportation system, generally serving as social infrastructure system. During constructing bridge there are three main components of the bridge, and they are (substructure, superstructure, bearing), etc. this study is concern about the manual analysis and design of the bridge, it's built for the purpose of providing passage over the obstacles, generally something that can obstacle to cross otherwise.
\end{abstract}




\section{INTRODUCTION}

Bridges are the structures that spans are horizontally between the support, and the function of the bridge is to carry vertical loads, so the vertical loads can be pointed or distributed load. The prototypical bridge is quite simple for two supports holding up the beams. The support must be strong enough to carry the structural loads. Spans always designed as short as possible; also, long spans are justified a good foundation. Bridges are important to every modern highway. But they are not seen or understood in the same way through everyone. There are types of bridges with different materials. Most of the people, when they are going to the job or other places, travel over the bridge everyday, so they don't realize the bridge, but they just look the roadway railing on the other side. The earliest bridge was stepping stones, and it was the simplest one bridge ever.

\section{THE SEVEN TYPES OF BRIDGE}

- There are seven types of bridge designsthat have been adapted and enhanced over the years.

- Although the truss bridges design is seen as the strongest suspension bridge design is the one which can give stretch utmost.

- The most expensive bridge is the suspension bridge with these days. There are different types of bridges, while typically, their structures can be to one of the seven main types.

\section{Beam Bridges}

This type of bridge is one of the simplest bridges. The traditional deck area of this bridge consists ofa stone slap, and the wood plank and the supports are sides by two beams running between the abutments or piers. Some of this bridge you will find other beams positioned in between the main beams also there are extra columns. The deck area where peoples or the vehicles travel there's underlying beams. This type of bridge referred simply supported beams. 


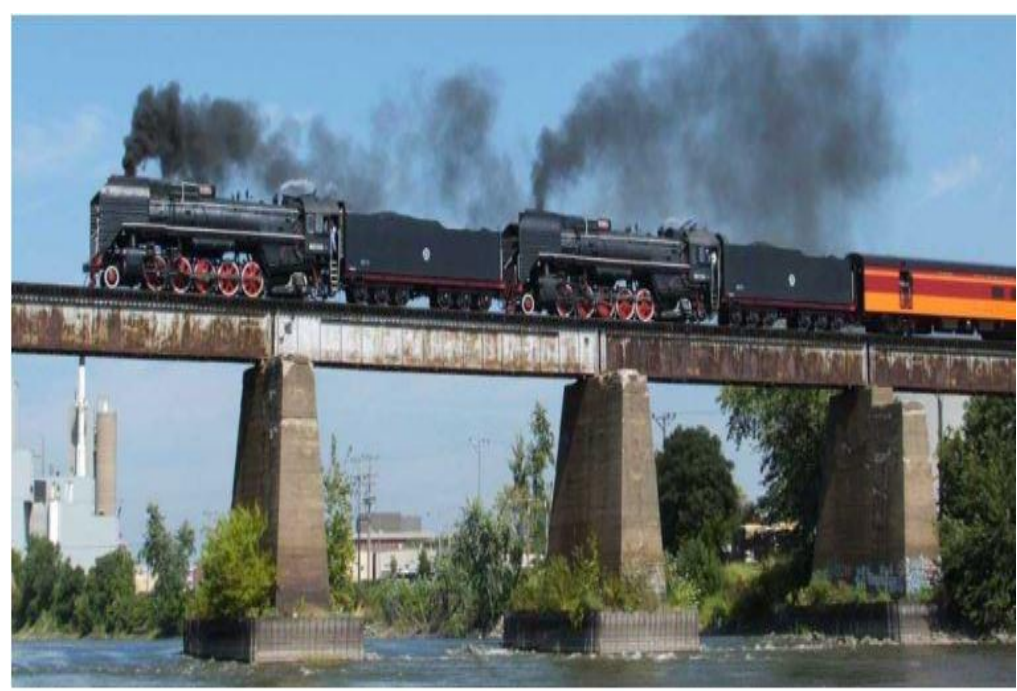

Fig 1: beam bridge

\section{Truss bridges}

Truss bridge has been used around recent centuries till now; these bridges are load-bearing structures that incorporate a truss in a highly efficient yet very simple design. You will see an array of different shapes of a simple truss bridge, but they are all incorporate triangular sections. So the role of these triangular truss elements is important inside of absorption loads in tension or compression. Therefore the mixture of tension and compression of the bridge is maintained, and the deck area will remain strong and safe.

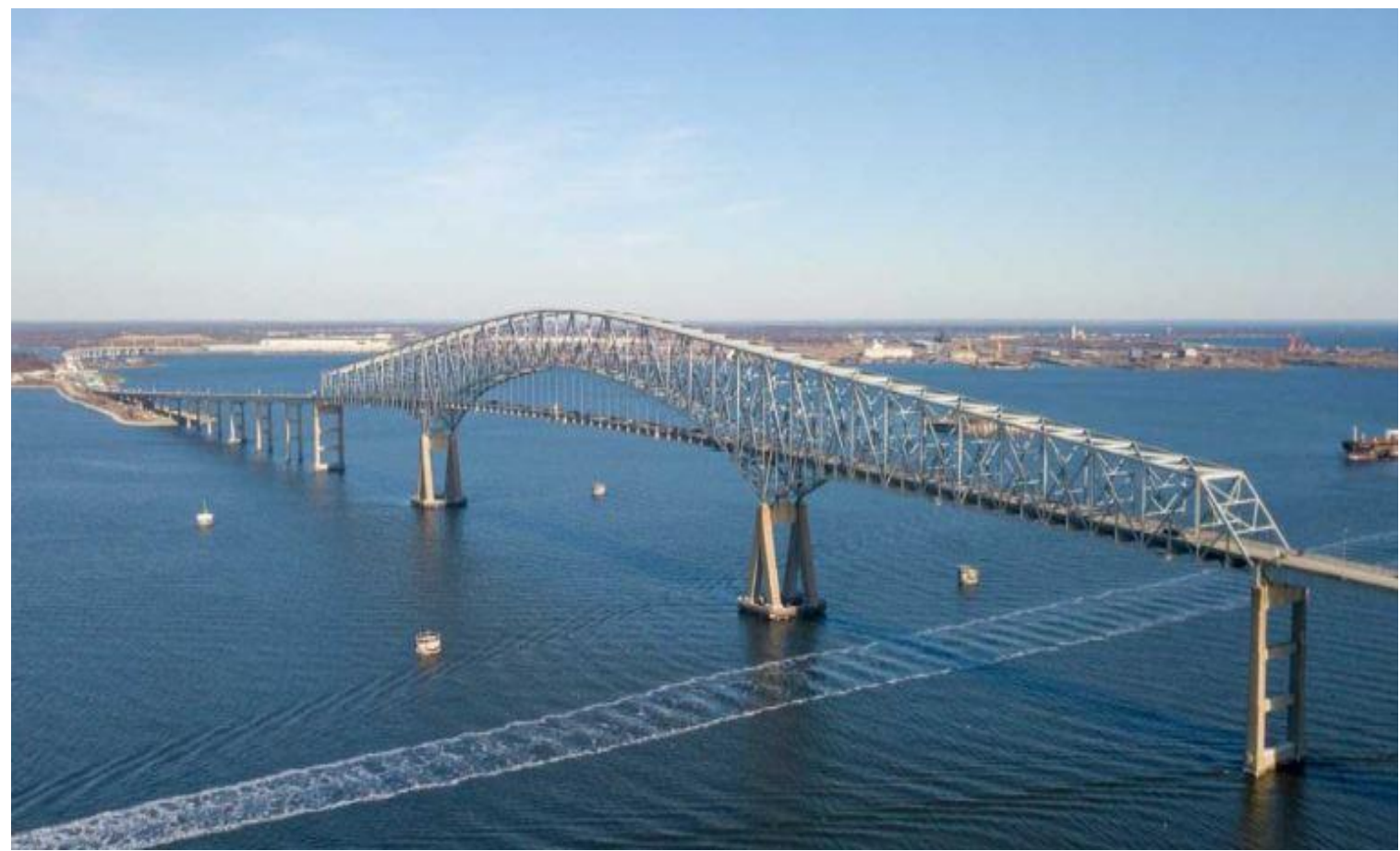

Fig 2: truss bridge 


\section{Cantilever bridges}

The first-time designed cantilever beam it was seen as a major engineering breakthrough. This type of bridge made from pre-stressed concrete or steel structure when used to accommodate the traffic. When you consider the horizontal beam making up cantilever arm are only supported from one side, it will look like a little dangerous. The two arms of the cantilever are connected through the suspended span, so it's effectively a centerpiece that has no direct support underneath. Therefore, this bridge is supported by diagonal bracing with horizontal beams as opposed to a typical vertical bracing. This type of bridge is very safe, secure; the design of this bridge is still using in this century.

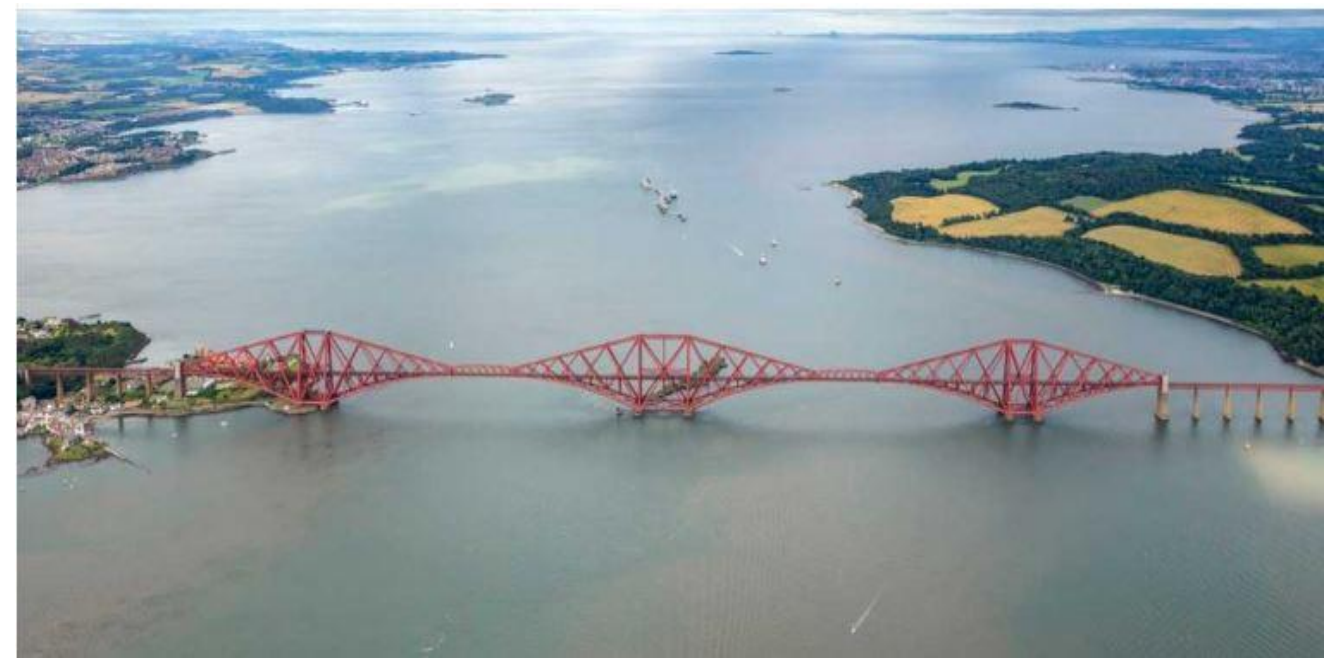

Fig 3: cantilever bridge

\section{Arch Bridges}

There are many different types of arch bridges, but they all have common think like the central element. So, each one of these bridges has an abutment, which is used to support the curved arch under the bridge. The most common type of arch bridge is a viaduct, a long arch bridge made on this. The lateral pressure acting on the span is transferred into the supporting abutments. It's very important to be this part a solid and well-founded. This type of bridge is very strong; he can carry the pedestrian and the vehicle load as well.

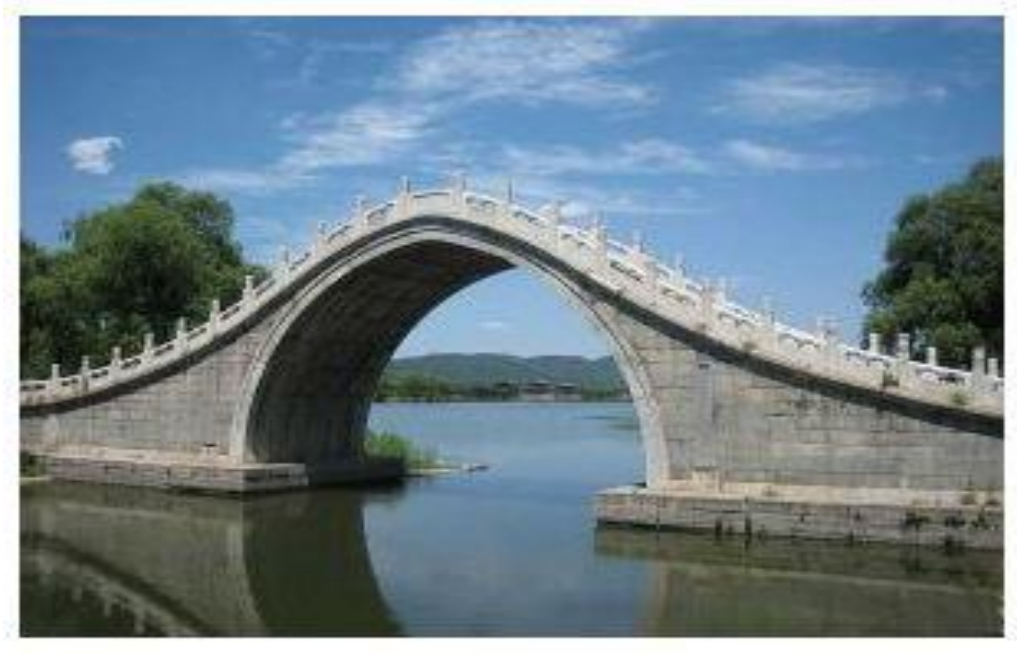

Fig 4:- arch bridge 


\section{Tied Arch Bridges}

This type of bridge is very beautiful, which incorporates an arch structure supported by vertical ties between the deck and the arch. The tips of the arch structure are connected at the bottom of the chord. This design is similar to the string of the bow. The downward pressure that is acting on the arch structure is transferred into the ties by tension and compression.

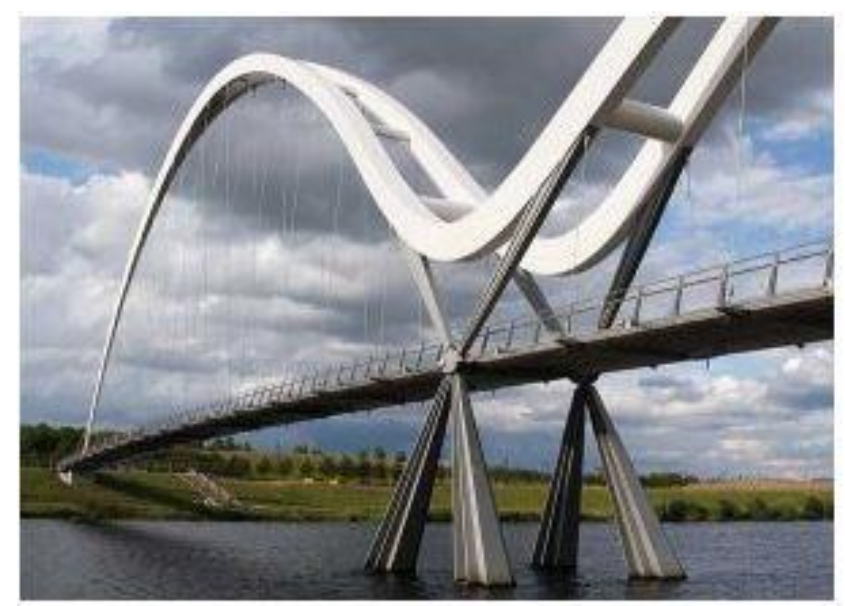

Fig 5: Tied Arch Bridges

\section{Suspension Bridges}

The suspension of this bridge looks very simple, but the design is extremely very effective. The deck area of the suspension bridge is a load-bearing element structure. This is held in a place by the vertical suspenders which are supporting the cables. The suspension cables extend out beyond each side of the bridge and are anchored firmly into the ground. It will depend upon the size of the bridge, but a number of towers will be installed to hold up the suspension cables. Any load applied to the bridge is transformed into tension across the suspension cables, which are an integral part of the structure. As there is some "give" in the suspension cables, this can translate into a slight, but measured bridge movement in difficult weather conditions.

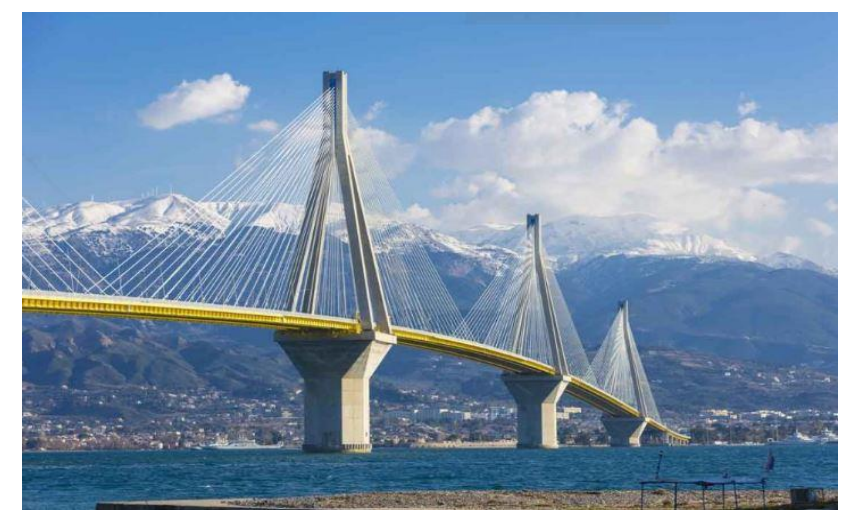

Fig 6: Suspension Bridges 


\section{Cable-stayed bridges}

This type of bridge depends upon towers or pylons, which are the loadbearing elements of the structure. The cables are connected from the deck below to the pylons, either directly from the top of the tower but at different points. Then when it connected differently positioned, this creates fan like patters. This type of bridge is intended for long-distance greater than those achieved with a cantilever bridge but less than the suspension bridge. The main problems of this bridge are that the central connection of the cables can be horizontal pace pressure on the deck, so the deck area needs to be reinforced to stand this pressure.

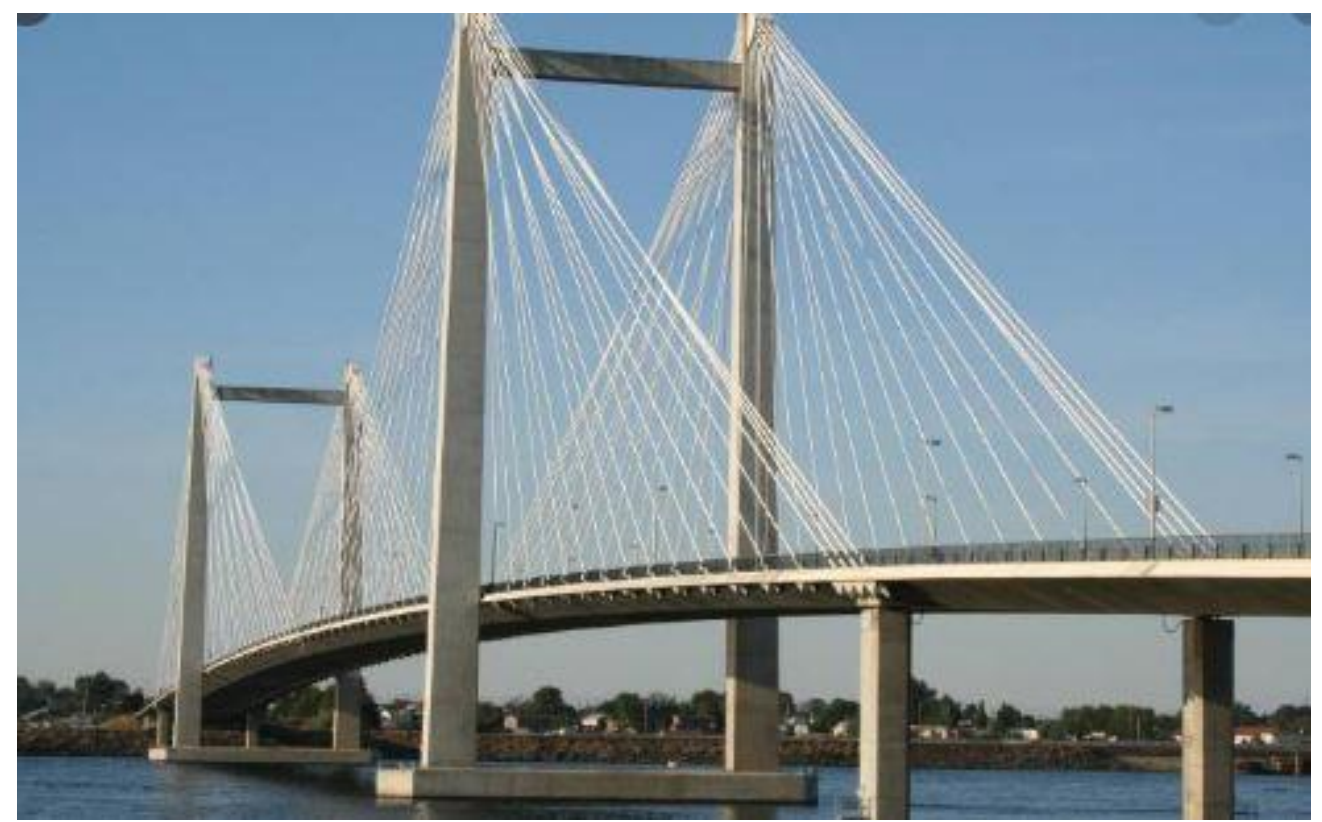

Fig 7: Cable-stayed bridges

\section{BASIC COMPONENT AND PARTS OF BRIGADE STRUCTURE}

Mostly bridge structures consist of the following component parts.

- Superstructure or decking component.

- Bearings.

- Substructure Components. 


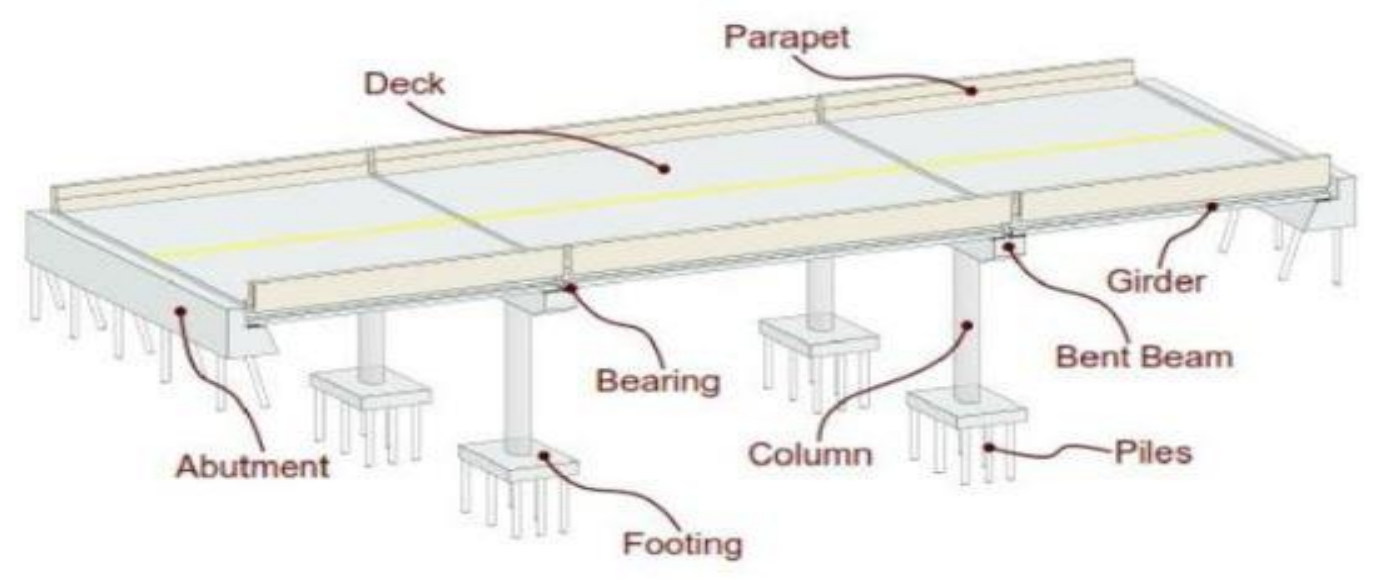

Fig8 : bridge component part

\section{SUPERSTRUCTURE COMPONENTS OF BRIDGES}

The upper part of the structure of the bridge component consistsof the truss, girder and the deck slap and etc. These component parts based on the bridge type, whether steel or concrete or composite. The superstructure of the bridge bears the load passing over it. This helps the transferring all force formed by the loads to the below substructures.

\section{Decks}

The deck slap is considering a road or rail surface of the bridge. Deck slaps are supported by the girders or the bigger beams thatare turn supported by the columns or the piers. The whole arrangement of the structure is supported with a deep foundation, mainly piles and cap arrangement.

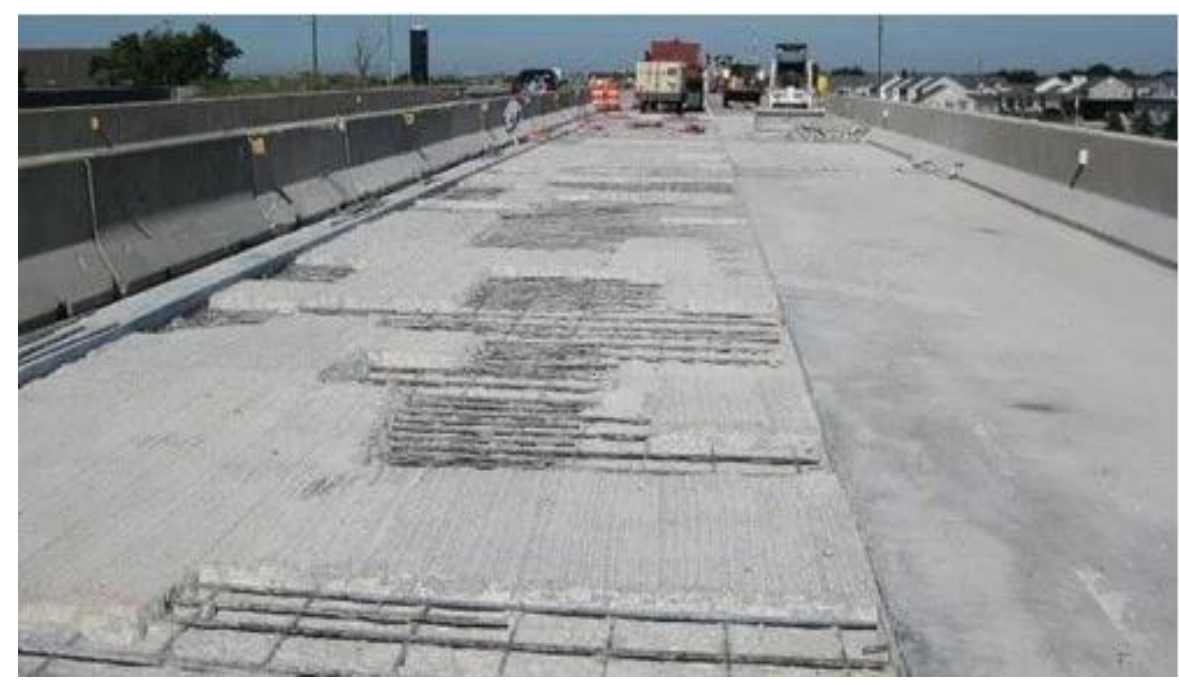

Fig 9: Deck slap 


\section{Bearings in Bridges}

The loads transmitted by the deck slap are properly and safely to the substructure with the help of the bearings. These parts are one of the components that help the bridge to transfer the superstructure loads to the substructure parts. The bearing in the bridge allows the longitudinal movement of the girders-this movementis coming due to the forces that are acting on the longitudinal direction. The forces due to the moving loads and the temperature change are the main causes of the longitudinal forces. The selection of the type of bearing it depends on a certain parameter like the; type of load acting, the geometrical plan, the clearance available, the displacement and the rotations, the extent maintenance.

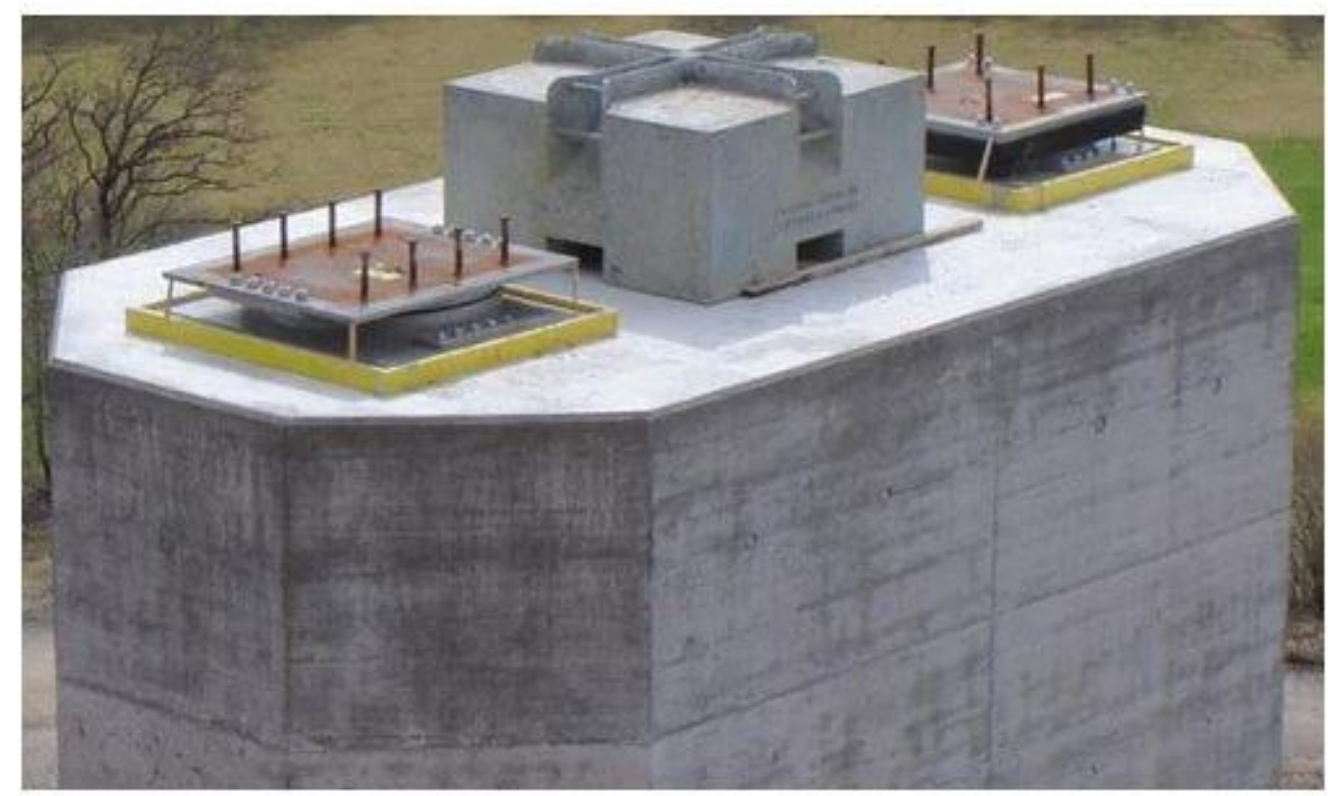

Fig 10: bearing of the bridge

\section{Substructure Components of Bridges}

The components involved in the substructure of bridges are:

1. Piers

2. Abutments

3. Wing Walls and the Returns

4. Foundation

\section{Piers}

The piers are the vertical members of the bridge used to support the deck slap; these members help the structure to hold up the deck slap and other vertical members that are above the slap.

- Load transmission to the foundation

- Resistance to the horizontal forces 
Piers are designed to resist the vertical loads coming from the slap. In the places that earthquakes happen, it's recommended to design the pier for the lateral loads. In general, the piers are constructed using concrete.

\section{TYPES OF PIERS IN BRIDGE CONSTRUCTION}

There are different types of piers based on the structural connectivity, the shape of the section, and the framing configuration.

- Based on the structural connectivity, the pier can be classified as monolithic or cantilevered.

- Based on the shape of the section pier can be classified as solid or hollow, hexagonal, round or octagonal or rectangular.

- Based on the framing configuration, the pier can be classified as single or multiple columns bent hammerhead or pier wall type.

\section{Abutments}

The abutment is the vertical structure used to retain the earth behind the built. The dead and live loads from the bridge superstructure is supported by the bridge abutment.

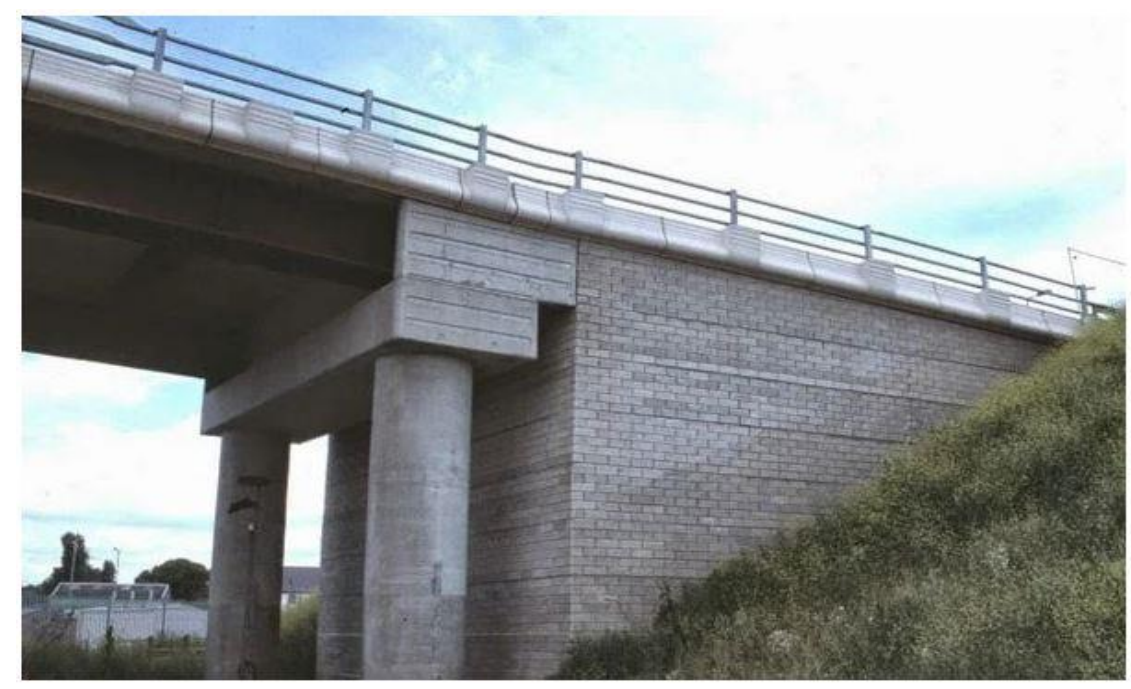

Fig 11: abutment

The abutments are also subjected to lateral pressures mainly from the approach embankment. The design loads on the abutment are mostly dependent on the:

- Type of abutment selected

- The sequence of construction 
The figure below shows the primary functions carried out by an abutment.

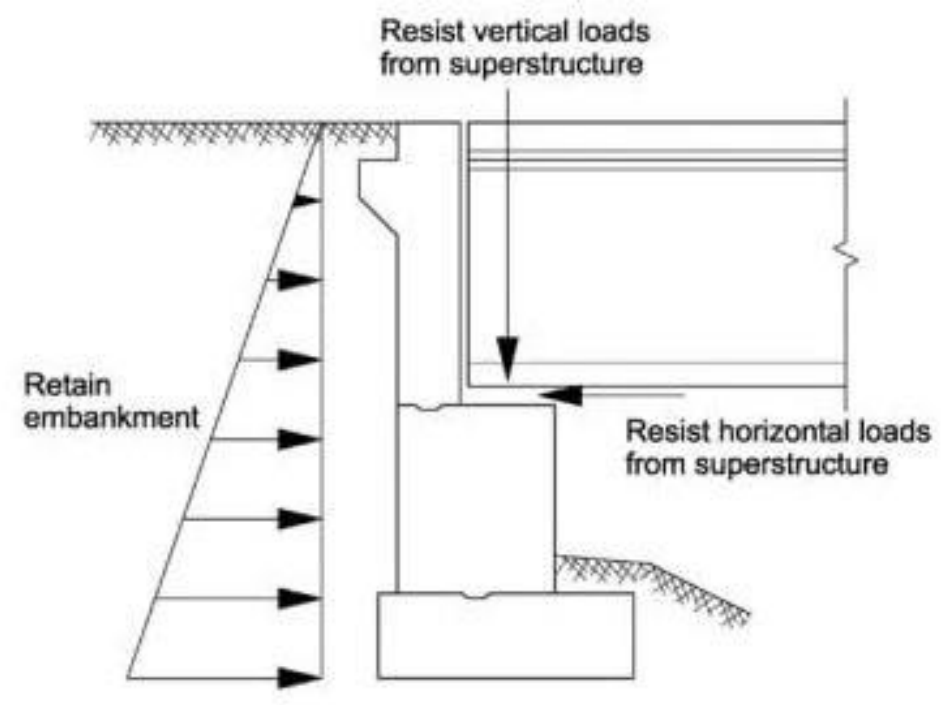

Fig 12: primary functions carried out by an abutment.

As seen from the above figure, the abutments have the design requirements similar to retaining walls as well as in pier construction. The abutments are primarily designed to resists the overturning and sliding. More focus is on the stability of the whole system. Special care has to be provided for the foundations of abutments. The abutment foundation must overcome the problems of differential settlement and excessive movements caused due to lateral forces or loads. The below figure shows the components of abutments.

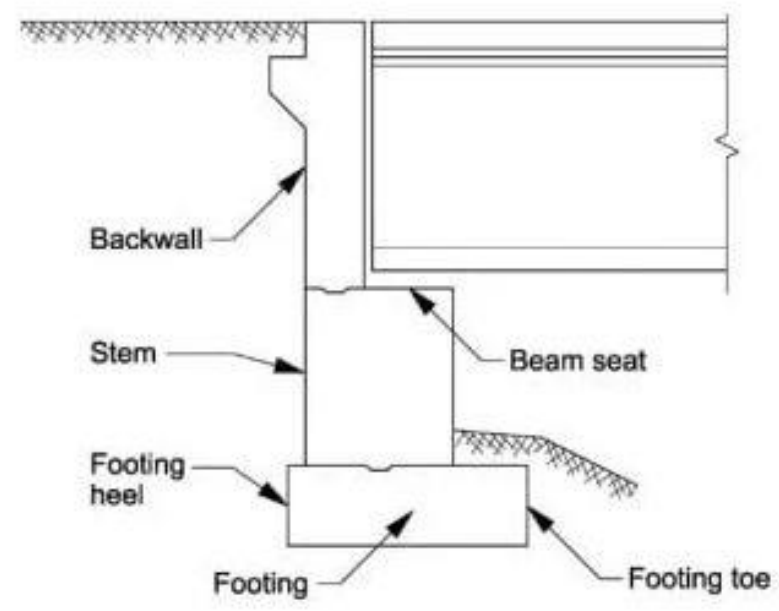

Fig 13: the components of abutments. 


\section{Wing Walls and Returns}

Structures constructed as an extension of the abutments to retain the earth present in the approach bank are called wing walls. This portion will otherwise have a natural angle of repose. These are retaining walls constructed adjacent to the abutments. This wall can be built either integrally or independently with the abutment wall.The rear of the wall must consider three design loads while designing. This includes:

- The earth pressure from the backfill

- The surcharge from the live loads or the compacting plant

- The hydraulic loads from the saturated soil conditions

The stability of the wing wall is mainly based on its resistance against the active earth pressures. The structural elements of the bridges are hereby designed and constructed to resist the earth pressures at rest.

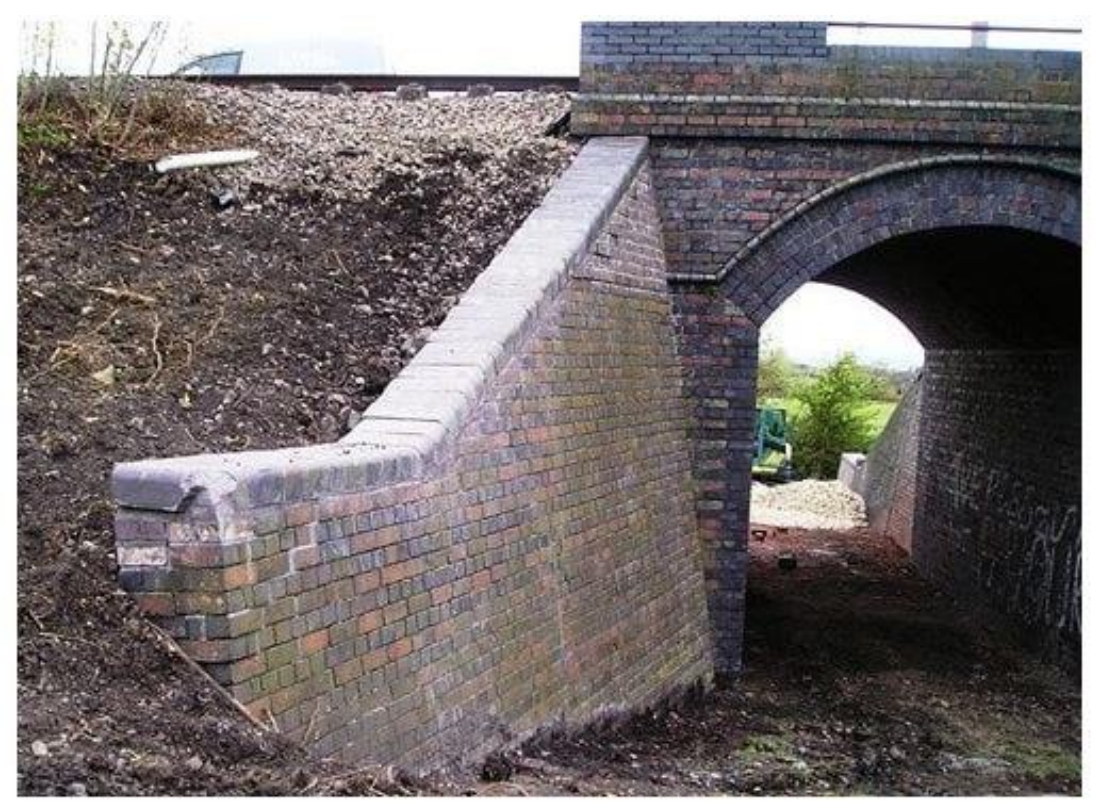

Fig 14: Wing Walls

\section{Parapets and Handrails/ Guard Rails or Curbs}

These components of bridges are not structural importance but provided for the safety concerns. These are provided above the decks. This will help in the prevention of the vehicle from falling off the bridge into the water body below or as a means for the separation of traffic streams. 


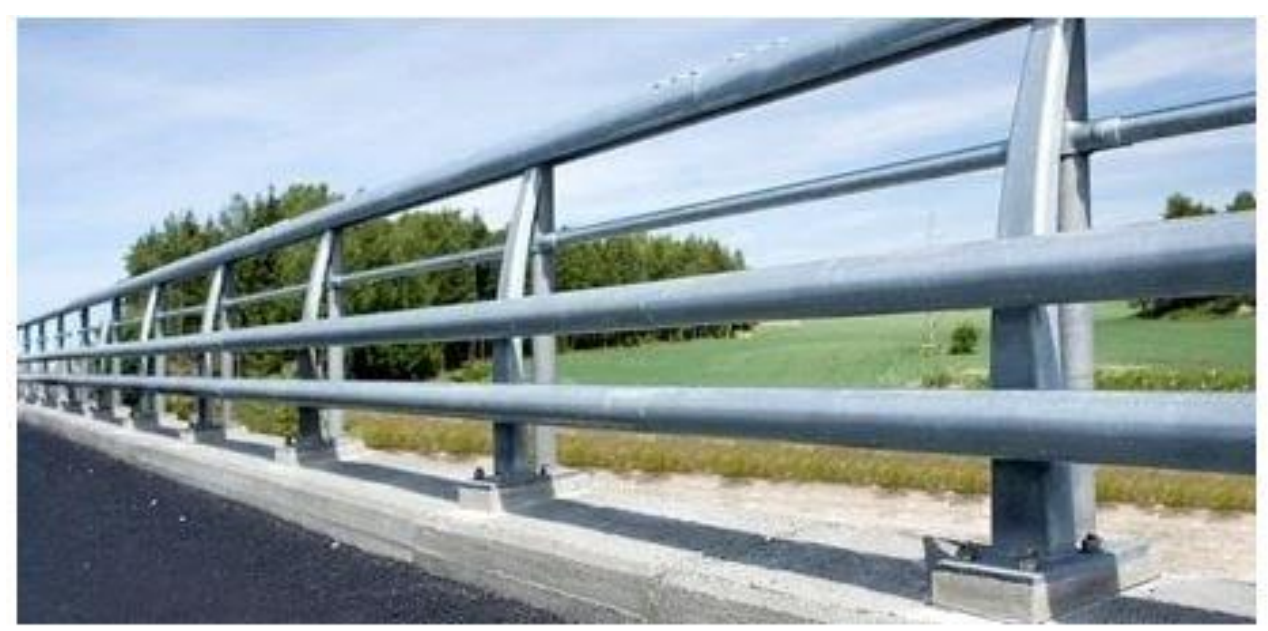

Fig 15: Handrails

\section{Foundation of Bridges}

Foundation are structures constructed to transmit the load from the piers, abutments, wing walls, and the returns evenly on the strata. The foundation provided for bridge structures isa deeply insufficient manner to avoid scouring due to the water movement or to reduce the chances of undermining.

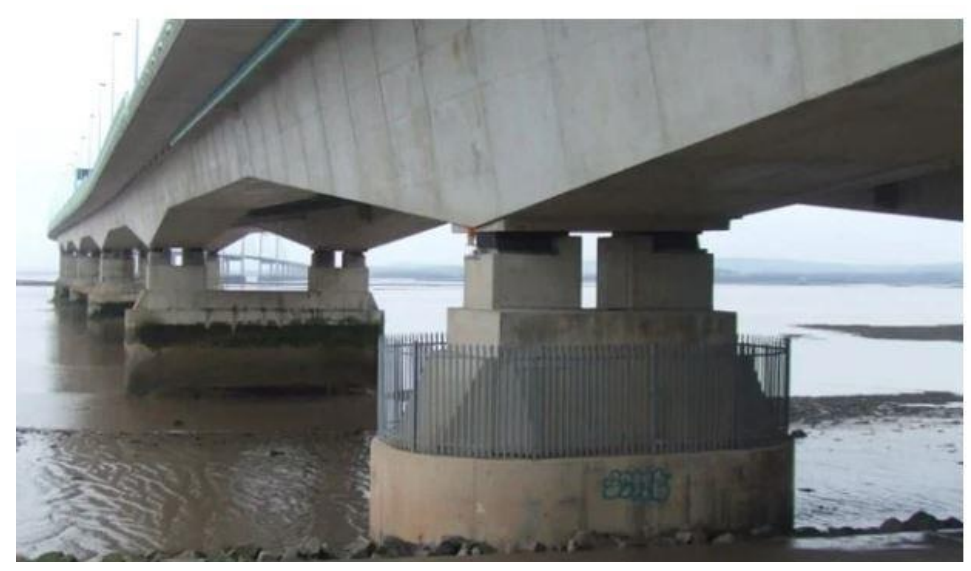

Fig 16: Foundation of Bridges 


\section{MANUALANALYSIS AND DESIGN OF THE BRIDGE}

IRC:6-2010
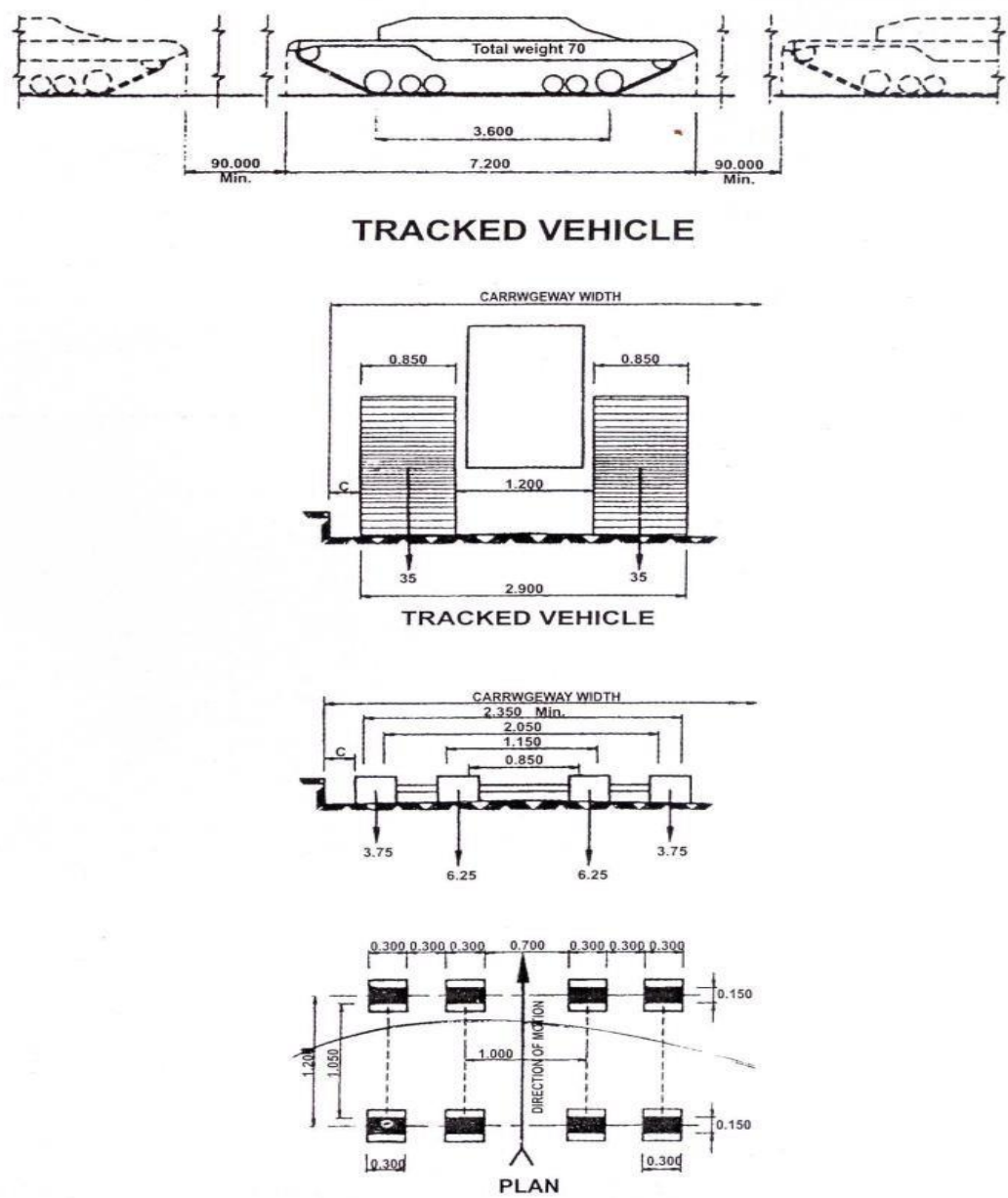

WHEELED VEHICLE

Fig. 1 Class AA Tracked and Wheeled Vehicles (Clause 204.1)

NOTES:

1) The nose to tail spacing between two successive vehicles shall not be less than $90 \mathrm{~m}$.

2) For multi-lane bridges and culverts, load combinations as given in Table 2 shall be adopted. Where IRC Class AA loading is specified it shall be used in place of Class $70 R$ but nose to tail distance shall be as specified in Note No.1.

9 
IRC:6-2010

3) The maximum loads for the wheeled vehicle shall be 20 tonne for a single axle or 40 tonne for a bogie of two axles spaced not more than $1.2 \mathrm{~m}$ centres.

4) The minimum clearance between the road face of the kerb and the outer edge of the wheel or track, C, shall be as under:

\begin{tabular}{lc}
\hline Carriageway width & Minimum value of $C(\mathrm{~m})$ \\
\hline Single - Lane Bridges & 0.3 \\
Upto width of $5.3 \mathrm{~m}$ & \\
$\begin{array}{l}\text { Multi-Lane Bridges } \\
\text { More than } 5.3 \mathrm{~m}\end{array}$ & 1.2 \\
\hline
\end{tabular}

5) Axle loads in tonne. Linear dimensions in metre.

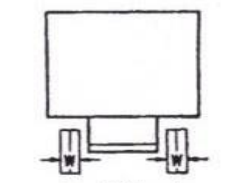

1.800 - 1

SECTION ON P-P

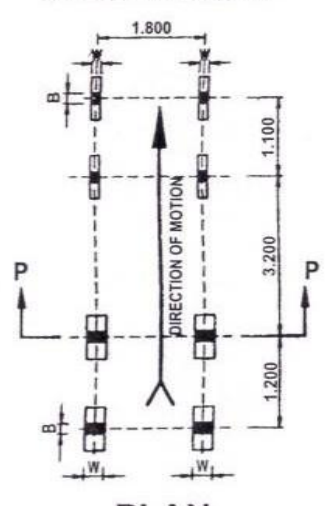

PLAN

DRIVING VEHICLE

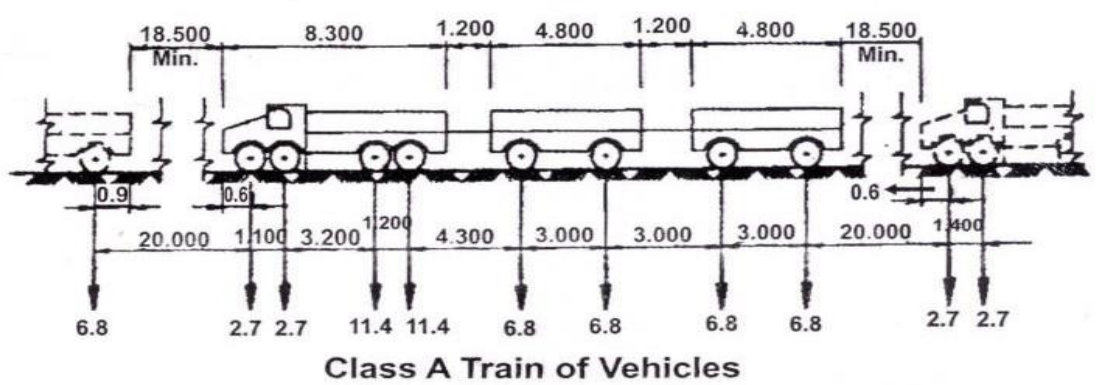

Fig. 2 Class 'A' Train of Vehicles (Clause 204.1)

10 
IRC:6-2010

2) No other live load shall cover any part of the carriageway when a train of vehicles (or trains of vehicles in multi-lane bridge) is crossing the bridge.

3) The ground contact area of the wheels shall be as under:-

\begin{tabular}{|l|c|c|}
\hline \multirow{2}{*}{$\begin{array}{l}\text { Axle load } \\
\text { (tonne) }\end{array}$} & \multicolumn{2}{|c|}{ Ground contact area } \\
\cline { 2 - 3 } & $B(\mathrm{~mm})$ & $W(\mathrm{~mm})$ \\
\hline 6.8 & 200 & 380 \\
\hline 4.1 & 150 & 300 \\
\hline 1.6 & 125 & 175 \\
\hline
\end{tabular}

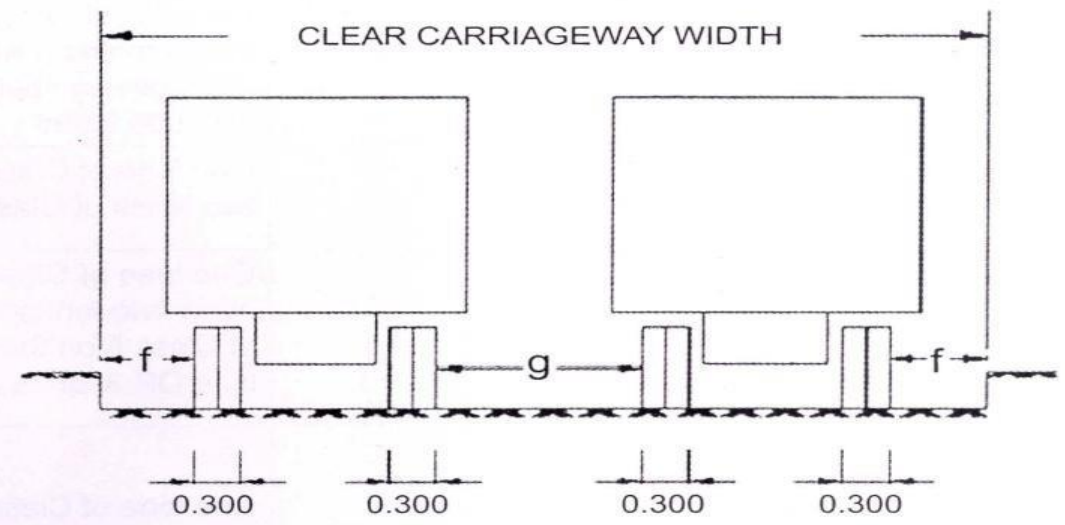

4) The minimum clearances, $f$, between outer edge of the wheel and the roadway face of the kerb and the minimum clearance, $\mathrm{g}$, between the outer edges of passing or crossing vehicles on multi-lane bridges shall be as given below:-

\begin{tabular}{|l|l|l|}
\hline Clear carriageway width & \multicolumn{1}{|c|}{$g$} & \multicolumn{1}{|c|}{$\mathrm{c}$} \\
\hline $5.5 \mathrm{~m}$ to $7.5 \mathrm{~m}$ & Uniformly increasing from & $150 \mathrm{~mm}$ for all carriageway \\
& $0.4 \mathrm{~m}$ to $1.2 \mathrm{~m}$ & widths \\
Above $7.5 \mathrm{~m}$ & $1.2 \mathrm{~m}$ & \\
\hline
\end{tabular}

5) Axle loads in tonne. Linear dimensions in metre.

204.1.4 Vehicles in adjacent lanes shall be taken as headed in the direction producing maximum stresses.

204.1.5 The spaces on the carriageway left uncovered by the standard train of vehicles shall not be assumed as subject to any additional live load unless otherwise specified in Table 2.

\subsection{Dispersion of Load through Fills of Arch Bridges}

The dispersion of loads through the fills above the arch shall be assumed at 45 degrees both along and perpendicular to the span in the case of arch bridges. 


\section{IMPACT}

208.1 Provision for impact or dynamic action shall be made by an increment of the live load by an impact allowance expressed as a fraction or a percentage of the applied live load.

\subsection{For Class A or Class B Loading}

In the members of any bridge designed either for Class A or Class B loading (vide Clause 204.1), this impact percentage shall be determined from the curves indicated in Fig.5. The impact fraction shall be determined from the following equations which are applicable for spans between $3 \mathrm{~m}$ and $45 \mathrm{~m}$.
i) Impact factor fraction for reinforced concrete bridges$$
=\frac{4.5}{6+L}
$$
ii) Impact factor fraction for steel bridges

$$
=\frac{9}{13.5+L}
$$

\section{HYDRAULIC DESIGN}

Design Discharge:

Catchment area $=522 \mathrm{sq} \cdot \mathrm{kms}$ Type of catchment $=$ Hilly - Good

Ryve ${ }^{e e}$ s coefficient $\mathrm{c}=2000$ as recommended by the technical committee for Cauvery basin.

By Ryve ${ }^{e e}$ Formula, Discharge $\mathrm{Q}=\mathrm{CA}^{2 / 3}$

Where, $Q=$ Maximum flood discharge $C=$ Ryve ${ }^{e c}$ coefficient $=2000$

$\mathrm{A}=$ Catchment area in square miles $=213$ sq.m $\mathrm{Q}=2000 * 213^{2 / 3}$

$=71331.78$ Cusecs

$\therefore$ Maximum flood discharge $=71331.78 \mathrm{Cusecs}=2020 \mathrm{~m}^{3} / \mathrm{sec}$ 
Linear Water Way: Discharge $=2020 \mathrm{~m}^{3} / \mathrm{sec}$ Linear water way $=\mathrm{C} \sqrt{ } \mathrm{Q}$

$=4.4 * \sqrt{ } 2020$

$=197.75 \mathrm{~m}=198.00 \mathrm{~mm}$

End $\operatorname{span}=19 * 2=38 \mathrm{~m}$ Intermediate span $=40 * 4=160 \mathrm{~m}$

\section{Afflux:}

Linear water way $=198.97 \mathrm{~m}$ Natural water way $=199 \mathrm{~m}$ Velocity of water, $V=1.85 \mathrm{~m} / \mathrm{s}$

Afflux $=\mathrm{a}=\left(\mathrm{V}^{2} / 2 \mathrm{~g}\right)\left[\left(\mathrm{L}^{2} / \mathrm{c}^{2} \mathrm{~L}_{1}^{2}\right)-1\right]$ Where $\mathrm{c}=0.9$ for Bell mouthed entry.

$\therefore$ Afflux $=\mathrm{a}=\left(1.85^{2} / 2 * 9.81\right)\left[\left(199^{2} / 0.9^{2} * 198.97^{2}\right)-1\right]$

$=0.0415 \mathrm{~m}$

\section{Scour Depth:}

For uniform scour,

Scour depth $=\mathrm{d}_{\max }=1.5 \mathrm{~d}^{\prime \prime} \mathrm{d}^{\mathrm{ee}}=\mathrm{d}\left[\frac{w}{\mathrm{~W}}\right]^{0.61}$

$\mathrm{d}=\left(1.21 \mathrm{Q}^{0.63}\right) /\left(\mathrm{f}^{0.33} \mathrm{w}^{0.6}\right)$

$=\left(1.21 * 2020^{0.63}\right) /\left(1.1^{0.33 * 199^{0.6}}\right)$

$=5.92 \mathrm{~m}$

$\mathrm{d}^{\mathrm{ee}}=5.92\left[\frac{199}{198.97}\right]^{0.61}$

$=5.92 \mathrm{~m}$

$\therefore \mathrm{dmax}=8.80 \mathrm{~m}$

For non-uniform scour,

$\mathrm{d}_{\max }=\mathrm{d}\left[\frac{w}{L}\right]^{1.56}$

$=5.92\left[\frac{199}{198.97}\right]^{1.56}$

$=5.92 \mathrm{~m}$ 


\section{STRUCTURAL DESIGN}

Preliminary Data:

Total span of the Bridge $=198.00 \mathrm{~m}$ Width of the road $=7.5 \mathrm{~m}$

Kerbs on each side $=600 \mathrm{~mm}$ Footpath on either side $=1.5 \mathrm{~m}$ Grade of concrete $=\mathrm{M} 20$

Loss ratio $=0.80$

Spacing of Cross- girder $=5 \mathrm{~m}$

Grade of steel $=$ Fe $415-$ HYSD bars Depth of slab $=250 \mathrm{~mm}$

Thickness of wearing coat $=80 \mathrm{~mm}$

Live load - Class AA tracked vehicle - 700kN

\section{ENDSPAN:}

\section{Design of Interior Panel:}

a) Bendingmoments:

Dead weight of slab $=(1 * 1 * 0.25 * 24)=6.00 \mathrm{kN} / \mathrm{m}^{2}$

Dead weight of wearing coat $=(0.008 * 22)=1.76 \mathrm{kN} / \mathrm{m}^{2}$ Total dead load $=7.76 \mathrm{kN} / \mathrm{m}^{2}$

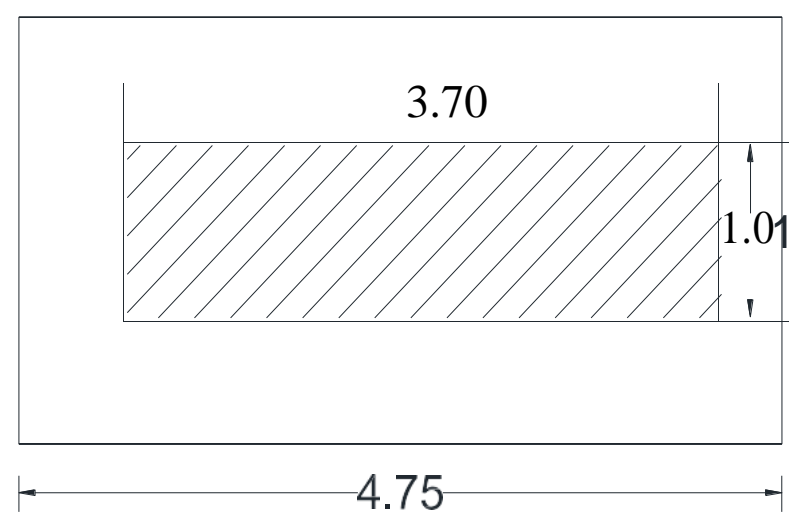

Position of IRC Class AA wheel load for maximum Bending moment

$\mathrm{U}=\mathrm{B}+2 \mathrm{t} \mathrm{V}=\mathrm{L}+2 \mathrm{t}$

$\mathrm{U}=(0.85+2 * 0.08)=1.01$

$\mathrm{V}=(3.6+2 * 0.08)=3.76$ 
$\mathrm{u} / \mathrm{B}=1.01 / 2.5=0.404$

$\mathrm{V} / \mathrm{L}=3.76 / 4.75=0.792$

From Pigeaud ${ }^{\text {ee }}$ c curve, $\mathrm{K}=\mathrm{B} / \mathrm{L}=0.53 \mathrm{~m}_{1}=0.095$ and $\mathrm{m}_{2}=0.02 \mathrm{M}_{\mathrm{B}}=\mathrm{w}\left(\mathrm{m}_{1}+0.15 \mathrm{~m}_{2}\right)$

$=350(0.095+0.15 * 0.02)$

$=34.30 \mathrm{kN}-\mathrm{m}$

As the slab is continuous, Design $\mathrm{BM}=0.8 \mathrm{MB}$

Design BM including impact and continuity factor

$=1.25 * 0.8 * 34.3$

$=34.30 \mathrm{kN}-\mathrm{m}$

$\mathrm{M}_{\mathrm{L}}=\mathrm{w}\left(\mathrm{m}_{2}+0.15 \mathrm{~m}_{1}\right)$

$=350(0.02+0.15 * 0.095)$

$=11.98 \mathrm{kN}-\mathrm{m}$

\section{Shear Force:}

Dispersion in the direction of span,

$=0.85+2(0.08+0.25)$

$=1.51 \mathrm{~m}$

For maximum shear, load is kept such that the whole dispersion is within span. The load is kept at $1.51 / 2=0.755 \mathrm{~m}$ from the edge of the beam.

Effective width of slab $=k x[1-(x / L)]+b_{w}$ Clear length of panel $=4.75-0.2=4.55 \mathrm{~m}$

$\therefore \mathrm{B} / \mathrm{L}=4.55 / 2.3=1.978$

From table, (IRC 21-2000) value of $\mathrm{k}$ for continuous slab is $=2.6$ Effective width of slab,

$=2.6 * 0.755[1-(0.755 / 2.3)]+[3.6+(2 * 0.08)]$

$=5.079 \mathrm{~m}$ 
Load per meter width $=350 / 5.079$

$=70 \mathrm{kN}$

Shear force per meter width $=70(2.3-0.755) / 2.3$

$=47.00 \mathrm{kN}$

Shear force with impact $=1.25 * 47.0=58.75 \mathrm{kN}$

\section{Dead Load BM And Shear Force:}

Total dead load $=7.76 \mathrm{kN} / \mathrm{m}^{2}$

Total dead load on panel $=2.5 * 4.75 * 7.76$

$$
=92.15 \mathrm{kN}
$$

For $\mathrm{UDL} \mathrm{U} / \mathrm{B}=1$ and $\mathrm{V} / \mathrm{L}=1 \mathrm{~K}=\mathrm{B} / \mathrm{L}=2.5 / 4.75=0.5261 / \mathrm{K}=1.9$

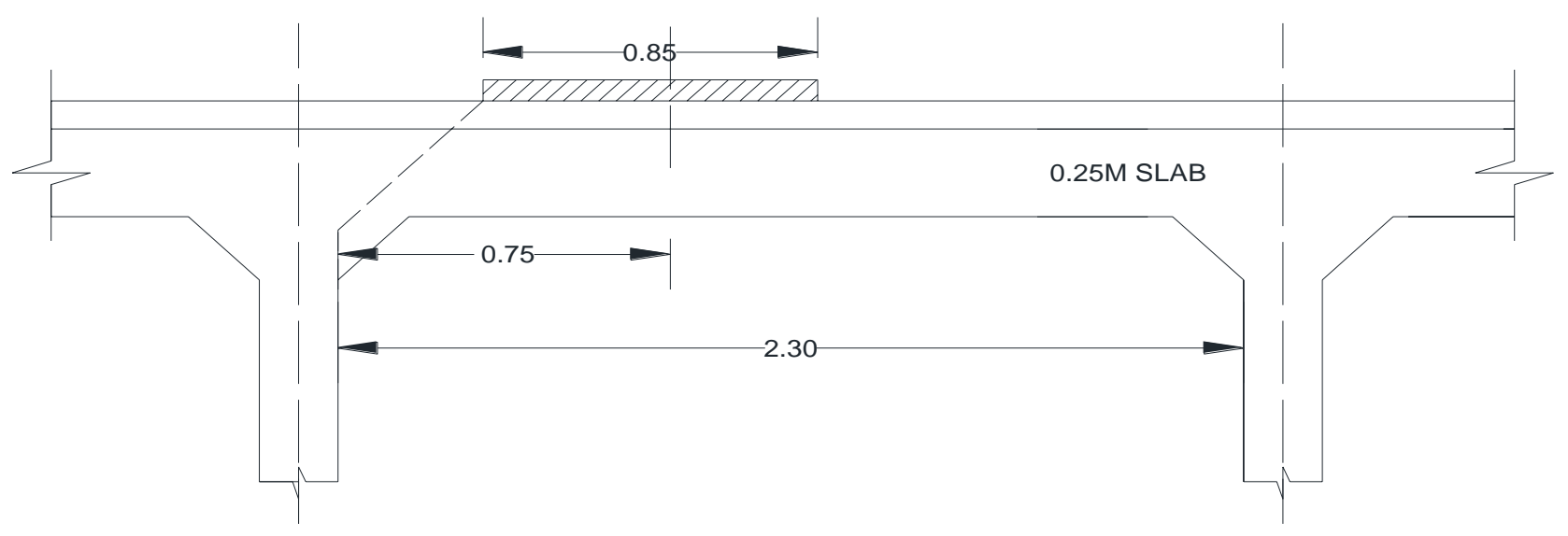

Position Of Wheel Load For Maximum Shear

From Pigeaud ${ }^{\text {ee }}$ curve, $\mathrm{m}_{1}=0.049$ and $\mathrm{m}_{2}=0.01$

$\mathrm{M}_{\mathrm{B}}=92.15\left(0.049+0.15^{*} .01\right)=4.65 \mathrm{kN}-\mathrm{m} \mathrm{M}_{\mathrm{L}}=92.15(0.01+0.15 * 0.049)=1.60 \mathrm{kN}-\mathrm{m}$

Design $\mathrm{BM}$ including continuity factor, $\mathrm{M}_{\mathrm{B}}=0.8 * 4.65=3.72 \mathrm{kN}-\mathrm{m} \mathrm{M}_{\mathrm{L}}=0.8 * 160=1.28 \mathrm{kN}-\mathrm{m}$

Dead load shear force $=\mathrm{w} 1 / 2$

$=\left(7.76^{*} 2.3\right) / 2$

$=8.92 \mathrm{kN}$ 
Design moments and shear forces: Total $\mathrm{M}_{\mathrm{B}}=34.3+3.72=38.02 \mathrm{kN}-\mathrm{m} \mathrm{M}_{\mathrm{L}}=11.98+1.28=13.26 \mathrm{kN}-\mathrm{m}$

\section{Design of Slab Section and Reinforcement:}

Effective depth, $d=\sqrt{ }(\mathrm{M} / \mathrm{Qb})$

$=\sqrt{ }\left(38.02 * 10^{6}\right) /(0.762 * 1000)$

$=223.37 \mathrm{~mm}$

$d_{\text {provided }}>d_{\text {efective. }}$ Hence ok.

Ast $=\mathrm{M} /\left(\sigma_{\mathrm{st}} * \mathrm{j} * \mathrm{~d}\right)$

$=\left(38.02 * 10^{6}\right) /(200 * 0.96 * 300)$

$=792 \mathrm{~mm}^{2}$

Use 12mm dia. Bars @150mm c/c

Center to center spacing $=\left[1000^{*}\left(\pi^{*} 12^{2}\right) / 4\right] / 792$

$=142.80 \mathrm{~mm}$

$=150 \mathrm{~mm} \mathrm{Ast}=\left[1000 *\left(* 12^{2}\right) / 4\right] / 150$

$=755 \mathrm{~mm}^{2}$

$\pi$

Effective depth for long span using 10mm dia.,

$=250-6-5$

$=239 \mathrm{~mm}$

Ast $=\left(13.26^{*} 10^{6}\right) /(200 * 0.96 * 239)$

$=288.96 \mathrm{~mm}^{2}=300 \mathrm{~mm}^{2}$ 
But minimum reinforcement using HYSD bars according o IRC $18-2000$ is $0.15 \%$ of cross section area. Hence,

Ast $=0.0015 * 1000 * 250$

$=375 \mathrm{~mm}^{2}$

\section{Check For Shear Stress:}

Nominal shear stress $=\tau_{\mathrm{v}}=\mathrm{V} / \mathrm{bd}$

$=\left(58.75 * 10^{3}\right) /(1000 * 250)$

$=0.235 \mathrm{~N} / \mathrm{mm}^{2}$

At support section, Ast $=755 \mathrm{~mm}^{2}$

Hence, $(100$ Ast $) / \mathrm{bd}=(100 * 755) /(1000 * 250)=0.302$

For $\mathrm{M}_{20}$ concrete, $\tau_{\mathrm{c}}=0.22 \mathrm{~N} / \mathrm{mm}^{2}$ (from table 12B IRC 21-2000) For overall depth $300 \mathrm{~mm}, \mathrm{~K}=1.01$ (From table 12C IRC:21-2000)

$\therefore$ Permissible shear stress in concrete $\mathrm{slab}=\mathrm{K} \tau_{\mathrm{c}=1.10 * 0.22}$

$=0.235 \mathrm{~N} / \mathrm{mm}^{2}>\tau_{\mathrm{v}=} 0.19 \mathrm{~N} / \mathrm{mm}^{2}$

Hence the shear stresses are within the safe permissible limits.

\section{Design of Longitudinal Girders:}

Reaction Factors:

Using Courbon"s theory, the IRC class AA loads are arranged for maximum eccentricity as shown in fig.

Reaction factor for outer girder $\mathrm{A}$ is, $\mathrm{R}_{\mathrm{A}}=\left(\sum \mathrm{w}_{1} / \mathrm{n}\right) *\left[1+\left(\operatorname{nex}_{1}\right) /\left(\Sigma \mathrm{x}^{2}\right)\right]$

$\left.=2 \mathrm{w}_{1} / 4\right) *\left[1+(4 * 1.1 * 3.75) /\left\{\left(2 * 3.75^{2}\right)+\left(2 * 1.25^{2}\right)\right\}\right]$

$=\underline{0.764} \mathrm{w}_{1}$ 


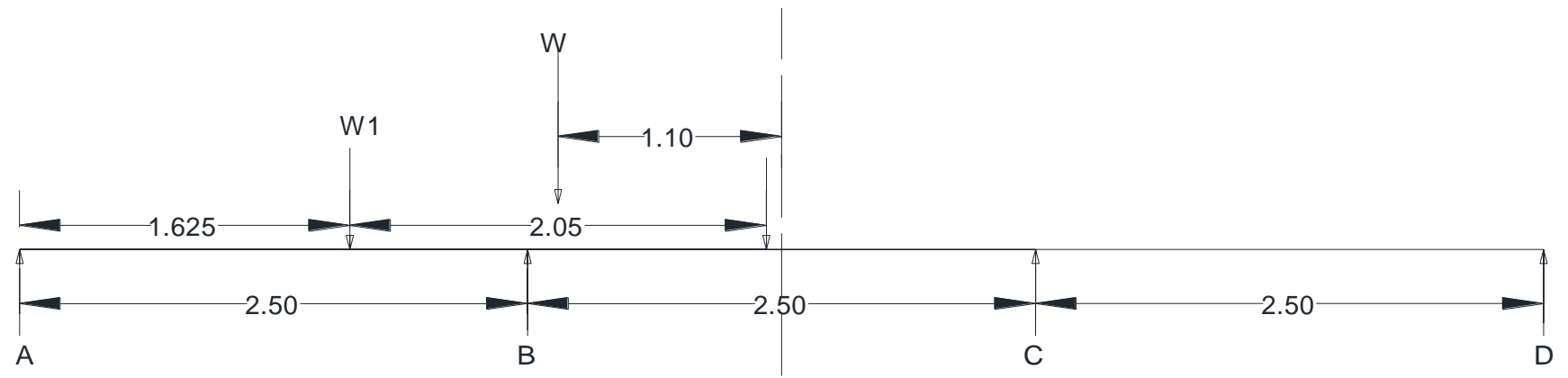

Transverse Disposition of IRC Class AA Tracked Vehicle

Reaction factor for inner girder B is,

$\left.\mathrm{R}_{\mathrm{B}}=2 \mathrm{w}_{1} / 4\right) *\left[1+\left(4 * 1.25^{*} 1.1\right) /\left\{\left(2 * 3.75^{2}\right)+\left(2 * 1.25^{2}\right)\right\}\right]$

$=\underline{0.588} \mathrm{w}_{1}$

If $\mathrm{w}_{1}=0.5 \mathrm{w} \mathrm{R}_{\mathrm{A}}=0.764 * 0.5 \mathrm{w}$

$=0.382 \mathrm{w}$

$\mathrm{R}_{\mathrm{B}}=0.588 * 0.5 \mathrm{w}$

$=0.294 \mathrm{w}$

\section{Dead Load From Slab Per Girder:}




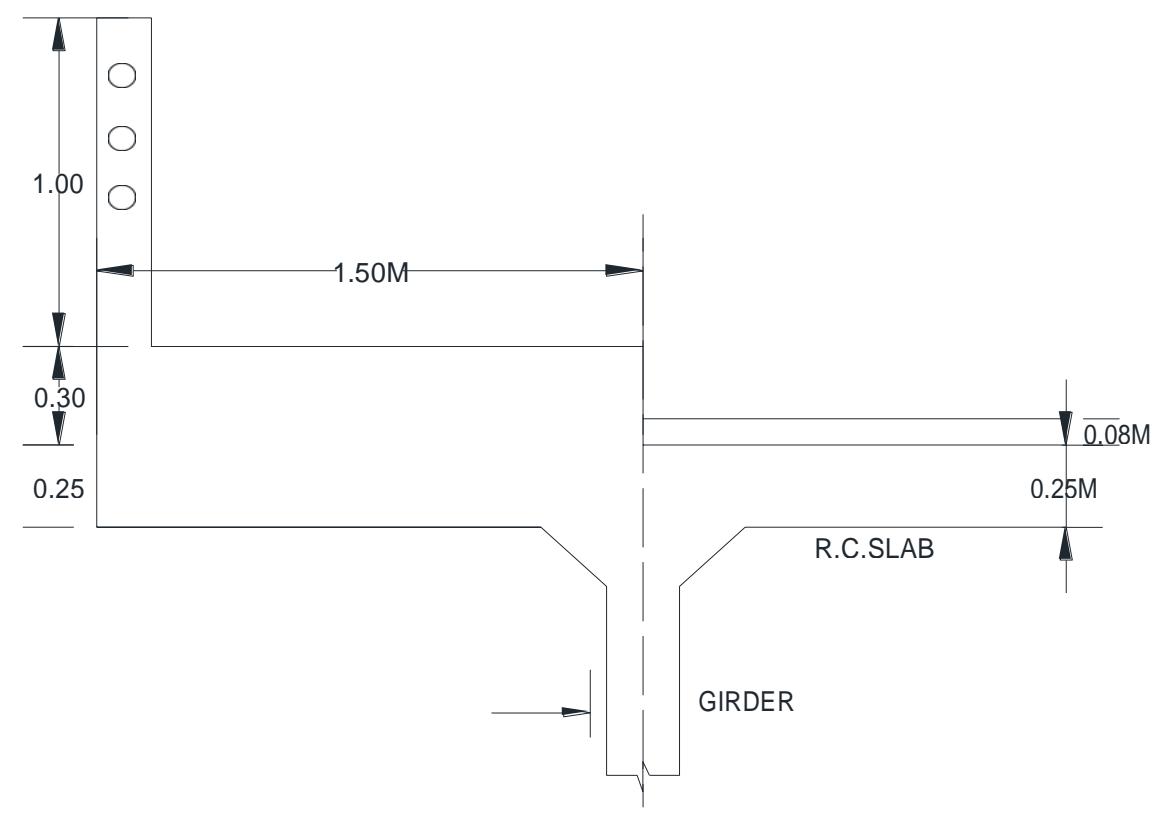

Weight of ,

Parapet railing (lump sum) $=0.92 \mathrm{kN} / \mathrm{m}$

Foot path and kerb $=0.3 * 1.5 * 24=10.08 \mathrm{kN} / \mathrm{m}$ Deck slab $=0.25 * 1.5 * 24=20.72 \mathrm{kN} / \mathrm{m}$

Total load on deck slab $=[(2 * 20)+(7.76 * 7.25)]=97.70 \mathrm{kN} / \mathrm{m}$

It is assumed that the deck load is shared equally by all the four girders.

$\therefore$ Dead load per girder $=97.70 / 4=24.425 \mathrm{kN} / \mathrm{m}$

\section{Dead Load On Main Girder:}

Overall depth of girder $=1800 \mathrm{~mm}$

Dead weight of rib $=1.15 * 0.2 * 24=5.52 \mathrm{kN} / \mathrm{m}$

Dead weight of bottom flange $=(0.5 * 0.4 * 24)=4.80 \mathrm{kN} / \mathrm{m}$ Totalload $\quad=10.32 \mathrm{kN} / \mathrm{m}$

Weight of cross girder $=(0.2 * 1.25 * 24)=6 \mathrm{kN} / \mathrm{m}$

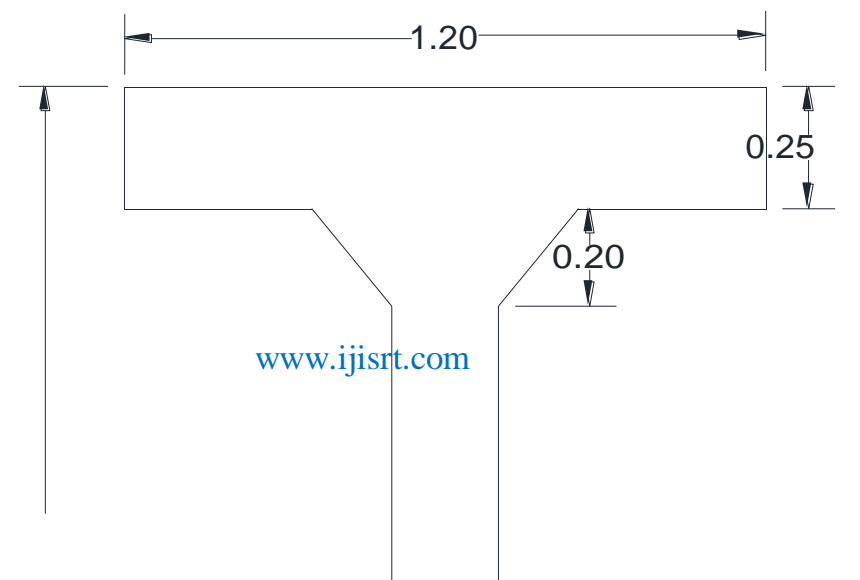



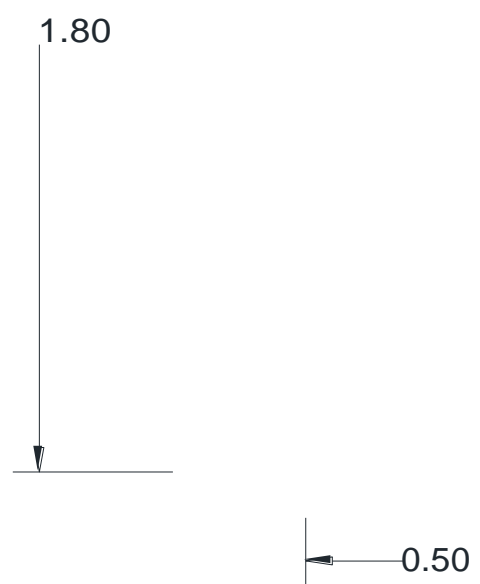

Cross section of Prestressed Concrete Girder Dead Load Moment and Shear In Main Girder:Reaction from deck slab on each girder $=24.55 \mathrm{kN} / \mathrm{m}$ Weight of cross girder $=6 \mathrm{kN} / \mathrm{m}$

Reaction on main girder $=\left(6^{*} 2.5\right)=15 \mathrm{kN} / \mathrm{m}$ Self weight of main girder $=10.32 \mathrm{kN} / \mathrm{m}$

Total dead load on girder $=(24.55+10.32)=34.87 \mathrm{kN} / \mathrm{m}$

The maximum dead load bending moment and shear force is computed using the loads shown in fig.

$\operatorname{Mmax}=\left[\left(0.125^{*} 34.87 * 19^{2}\right)+\left(0.25^{*} 15^{*} 19\right)+\left(15^{*} 9.5\right)+\left(15^{*} 4.75\right)\right.$

$=1858.51 \mathrm{kN}-\mathrm{m}$ 


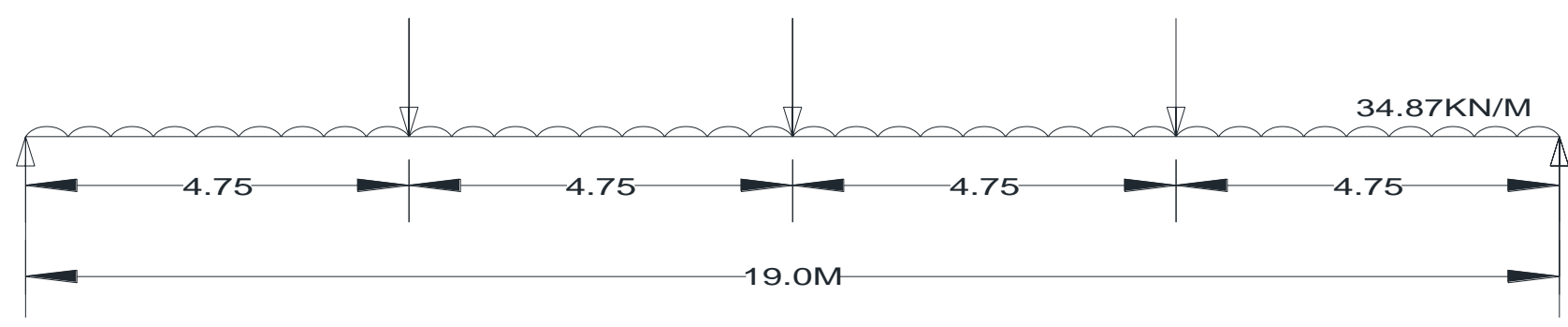

Dead Load on Main Girder

Dead load shear at support

$\operatorname{Vmax}=[(0.5 * 34.87 * 19)+(0.5 * 15 * 3)$

$=353.76 \mathrm{kN}$

Live Load Bending Moment in Girder:

Span of the girder $=19 \mathrm{~m}$ Impact factor $($ class AA $)=25 \%$

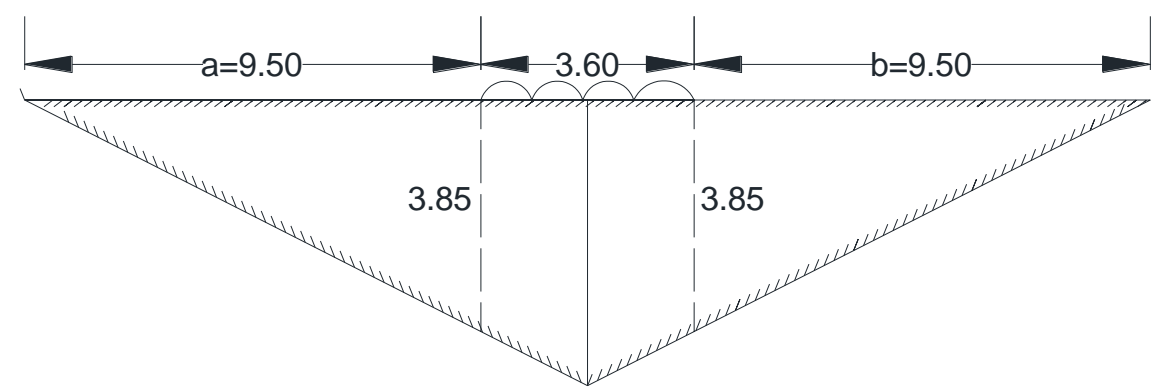

Influence Line Diagram for Bending Moment In Girder

Live load is placed centrally on the span Bending moment at center of span

$=0.5 *(3.85+4.75) * 700$

$=3010 \mathrm{kN}-\mathrm{m}$ 
Bending moment including impact and reaction factor for outer girder is, Live load bending moment $=$ $3010 * 1.25 * 0.382=1437.275 \mathrm{kN}-\mathrm{m}$

For inner girder, $\mathrm{BM}=3010 * 1.25 * 0.294=1106.175 \mathrm{kN}-\mathrm{m}$

Live Load Shear Forces in Girder:

For maximum live load shear in the girder, the IRC class AA loads are placed as, Reaction of $\mathrm{w}_{2}$ on girder B $=(350 * 0.45) / 2.5=63 \mathrm{kN}$

Reaction of $\mathrm{w}_{2}$ on girder $\mathrm{A}=(350 * 2.05) / 2.5=287 \mathrm{kN}$ Total load on girder $\mathrm{B}=350+63=413 \mathrm{kN}$

Maximum reaction in girder $\mathrm{B}=(413 * 18.2) / 19=395.61 \mathrm{kN}$

Maximum reaction in girder $\mathrm{A}=(287 * 18.2) / 19=274.92 \mathrm{kN}$

Maximum live load shear with impact factor in inner girder $=395.61 * 1.25=494.51 \mathrm{kN}$

Outer girder $=294.92 * 1.25=343.64 \mathrm{kN}$

Design Bending Moment and Shear Force:

\begin{tabular}{|c|c|c|c|c|}
\hline Bending Moment & Dead Load BM & Live load BM & Total BM & Unit \\
\hline Outer girder & 1858.51 & 1437.275 & 3295.785 & $\mathrm{kN}-\mathrm{m}$ \\
\hline Inner girder & 1858.51 & 1106.175 & 2964.685 & $\mathrm{kN}-\mathrm{m}$ \\
\hline \multicolumn{5}{|l|}{} \\
\hline Shear force & Dead load SF & Live load SF & Total SF & Unit \\
\hline Outer girder & 395.50 & 343.64 & 739.14 & $\mathrm{kN}$ \\
\hline Inner girder & 395.50 & 494.52 & 890.00 & $\mathrm{kN}$ \\
\hline
\end{tabular}




\section{PROPERTIES OF MAIN GIRDER SECTION:}

Cross sectional area $=(1200 * 250)+(1150 * 200)+(400 * 500)=73 * 10^{4} \mathrm{~mm}^{2}$

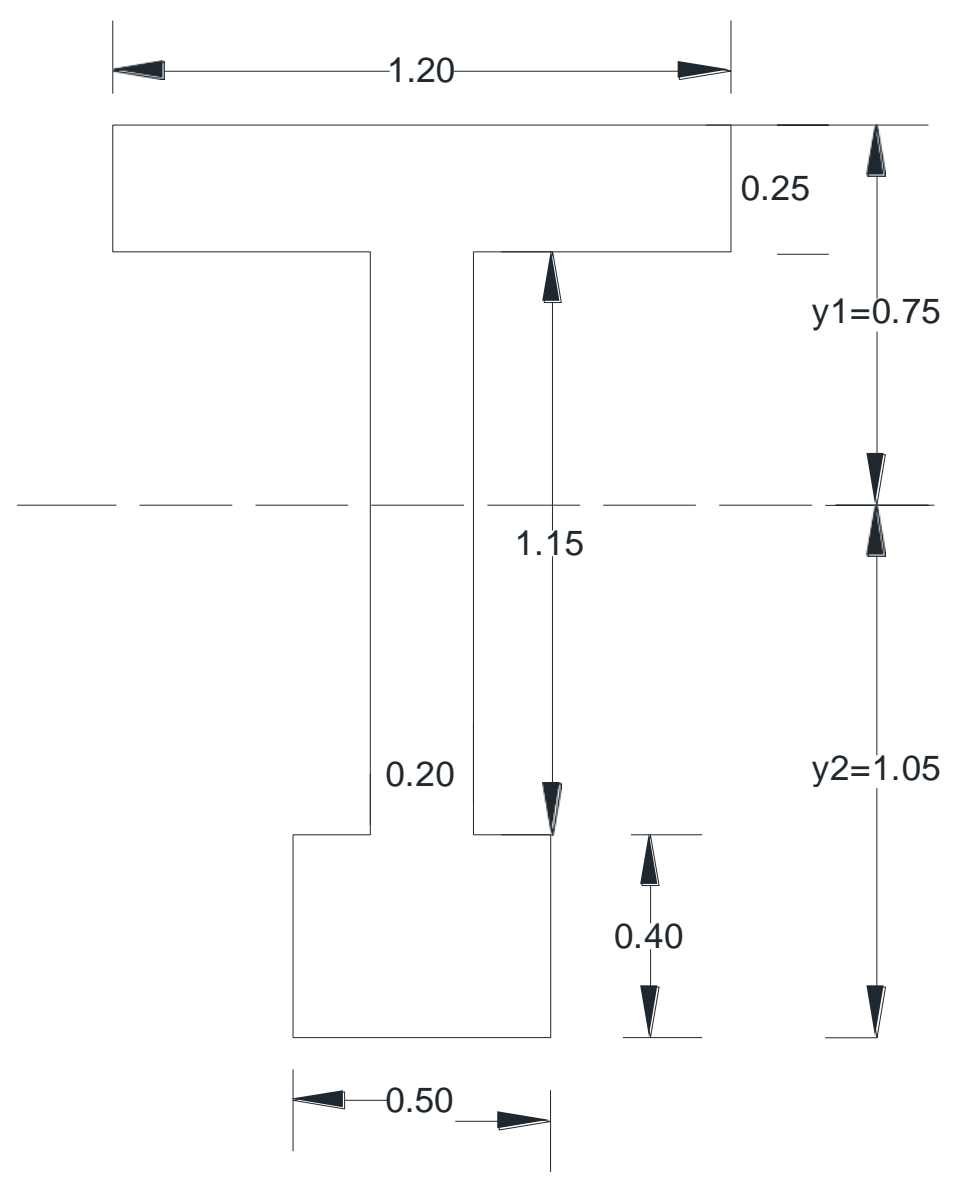

Cross Section Of Main Girder

To locate position of centroid, $\mathrm{Y}_{1}=\Sigma \mathrm{ay}^{1 /} / \Sigma \mathrm{a}$

$=750 \mathrm{~mm}$

$$
=\frac{1200 * 250) 125+(1150 * 200) 825+(500 * 400) 1600}{(1200 * 250)+(1150 * 200)+(500 * 400)}
$$

$\mathrm{Y}_{2}=1800-750=1050 \mathrm{~mm}$

Moment of inertia about axis of bending,

$\mathrm{I}=\left[\left\{\left(1200 * 250^{3}\right) / 12\right\}+(1200 * 250)(750-125)^{2}\right]+\left[\left\{\left(1150 * 200^{3}\right) / 12\right\}+(1150 * 200)(750-825)^{2}\right]+$ $\left[\left\{\left(500 * 400^{3}\right) / 12\right\}+(500 * 400)(750-1600)^{2}\right]$ 
$=2924 * 10^{8} \mathrm{~mm}^{4}$

$\mathrm{Z}_{\mathrm{t}}=\mathrm{I} / \mathrm{y}_{1}=3.89 * 10^{8} \mathrm{~mm}^{3} \mathrm{Z}_{\mathrm{b}}=\mathrm{I} / \mathrm{y}_{2=2} .78^{*} 10^{8} \mathrm{~mm}^{3}$

Check for Minimum Section Modulus:

$\mathrm{f}_{\mathrm{ck}}=50 \mathrm{~N} / \mathrm{mm}^{2}$

$\mathrm{f}_{\mathrm{ci}}=40 \mathrm{~N} / \mathrm{mm}^{2}$

$\mathrm{M}_{\mathrm{L}}=1437.275 \mathrm{kN}-\mathrm{m}$

$F_{b r}=\eta f_{c t}-f_{t w}$

$=0.8 * 18-0$

$=14.40 \mathrm{~N} / \mathrm{mm}^{2}$

$F_{t r}=f_{c w}-\eta f_{t t}$

$=16 \mathrm{~N} / \mathrm{mm}^{2}$

Inferior stress $=f_{i n f}=\left(f_{t w} / \eta\right)+\left(M_{D} / \eta Z_{b}\right)$

$=0+\left(3295.785^{*} 10^{6}\right) /\left(0.8 * 2.78 * 10^{8}\right)$

$=14.82 \mathrm{~N} / \mathrm{mm}^{2}$

Minimum section modulus required, $\mathrm{Z}_{\mathrm{required}}=\left[\mathrm{M}_{\mathrm{L}}+(1-\eta) \mathrm{M}_{\mathrm{G}}\right] / \mathrm{F}_{\mathrm{br}}$

$=\left[1437.275^{*} 10^{6}+(1-0.8) 1858.51 * 10^{6}\right] / 14.40$

$$
=1.25 * 10^{8} \mathrm{~mm}^{3}<2.78^{*} 10^{8} \mathrm{~mm}^{3}
$$

Hence the section provided is adequate. 


\section{Pre-Stressing Force:}

Assume a cover of $200 \mathrm{~mm}$

Maximum possible eccentricity e $=(1050-200)=850 \mathrm{~mm}$ Prestressing force is obtained as,

$\mathrm{P}=\left(\right.$ A. $\left.f_{\text {inf. }} Z_{b}.\right) /\left(Z_{b}+\right.$ A.e $)$

$=\left[\left(73 * 10^{\left.\left.4 * 14.82 * 2.78 * 10^{8}\right) /\left(2.78 * 10^{8}\right)+\left(73 * 10^{4} * 850\right)\right]}\right.\right.$

$=3393.177 * 10^{3} \mathrm{~N}$

$=3393 \mathrm{kN}$

Using Freyssinet system, anchorage type 7K-15 ( 7 strands of $15.2 \mathrm{~mm}$ diameter ) in $65 \mathrm{~mm}$ cab les duct, (IS:6006-1983)

Force in each cable $=(7 * 0.8 * 280.7)=1459 \mathrm{kN}$ No. of cables $=3393 / 1459=2.32=3$ no.s

Area of each strand $=140 \mathrm{~mm}^{2}$

Area of 7 strands in each canle $=7 * 140=980 \mathrm{~mm}^{2}$

Area of each strand in 3 cables $=A_{p}=3 * 980=2940 \mathrm{~mm}^{2}$ The cables are arranged at centre of span asshown

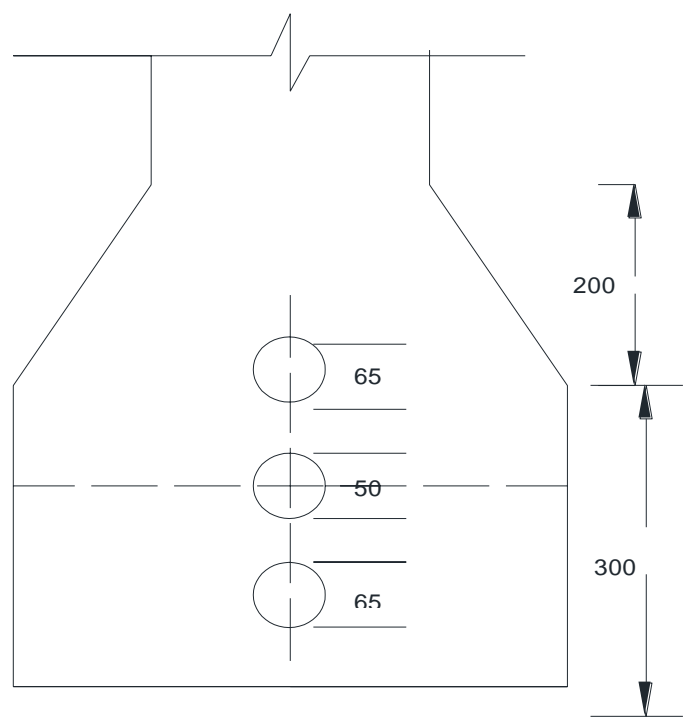

500

\section{Permissible Tendon Zone:}

At Support section, $\mathrm{e}=<\left(\mathrm{Z}_{\mathrm{b}} \cdot \mathrm{f}_{\mathrm{ct} /} \mathrm{P}\right)-\left(\mathrm{Z}_{\mathrm{b}} / \mathrm{A}\right)$

$=<\left[\left(2.78 * 10^{8 * 18)} /\left(3393 * 10^{3}\right)\right]-\left(2.78 * 10^{8}\right) /\left(0.73 * 10^{6}\right)\right.$ 
$=<1093.97 \mathrm{~mm}$

And $\mathrm{e}=>\left(\mathrm{Z}_{\mathrm{b}} \cdot \mathrm{f}_{\mathrm{tw} / \mathrm{\eta}} \mathrm{P}\right)-\left(\mathrm{Z}_{\mathrm{b}} / \mathrm{A}\right)$

$=>0-\left(2.78 * 10^{8}\right) /\left(0.73 * 10^{6}\right)$

$=-380 \mathrm{~mm}$

Check for Stresses:

For the centre of the span section, we have $\mathrm{P}=3393 \mathrm{kN}$

$\mathrm{e}=850 \mathrm{~mm}$

$\mathrm{A}=0.73^{*} 10^{6} \mathrm{~mm}^{2}$

$\mathrm{Z}_{\mathrm{t}}=3.89 * 10^{8} \mathrm{~mm}^{3}$

At transfer stage,

$\sigma_{\mathrm{t}}=\left[(\mathrm{P} / \mathrm{A})-\left(\mathrm{Pe} / \mathrm{Z}_{\mathrm{t}}\right)+\left(\mathrm{M}_{\mathrm{L}} / \mathrm{Z}_{\mathrm{t}}\right)\right]$

$=\left[\left(3393 * 10^{3}\right) /\left(73 * 10^{4}\right)-\left\{\left(3393 * 10^{3 * 850)} /\right.\right.\right.$

$\left.\left.\left(3.89 * 10^{8}\right)\right\}+\left(1437.275^{*} 10^{6}\right) /\left(3.89 * 10^{8}\right)\right]$

$=4.65-7.41-3.69$

$=0.93 \mathrm{~N} / \mathrm{mm}^{2}$

$\sigma_{\mathrm{b}}=\left[(\mathrm{P} / \mathrm{A})+\left(\mathrm{Pe} / \mathrm{Z}_{\mathrm{b}}\right)-\left(\mathrm{M}_{\mathrm{L}} / \mathrm{Z}_{\mathrm{b}}\right)\right]$

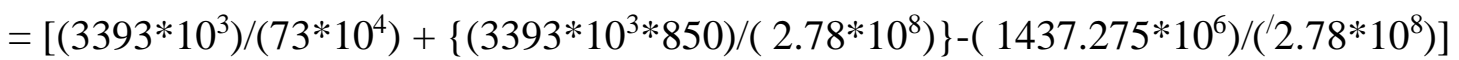

$=4.65+10.37-5.17$

$=9.85 \mathrm{~N} / \mathrm{mm}^{2}$ 
At working load stage,

$\sigma_{t}=\left[(n \mathrm{P} / \mathrm{A})-\left(\mathrm{nPe} / \mathrm{Z}_{\mathrm{b}}\right)+\left(\mathrm{M}_{\mathrm{D}} / \mathrm{Z}_{\mathrm{t}}\right)\right]$

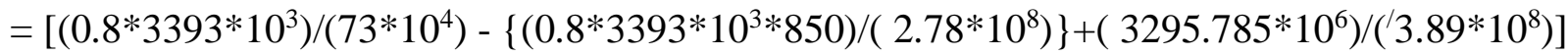

$=3.72-8.3+8.47$

$=3.89 \mathrm{~N} / \mathrm{mm}^{2}$

$\sigma_{\mathrm{b}}=\left[(\mathrm{nP} / \mathrm{A})+\left(\eta \mathrm{Pe} / \mathrm{Z}_{\mathrm{t}}\right)-\left(\mathrm{M}_{\mathrm{D}} / \mathrm{Z}_{\mathrm{b}}\right)\right]$

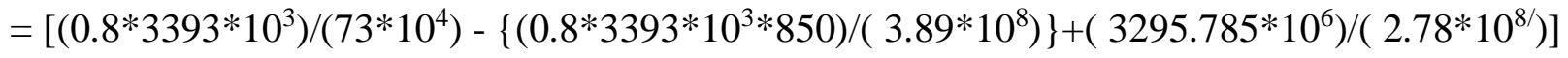

$=3.72+5.93-11.85$

$=-2.20 \mathrm{~N} / \mathrm{mm}^{2}$

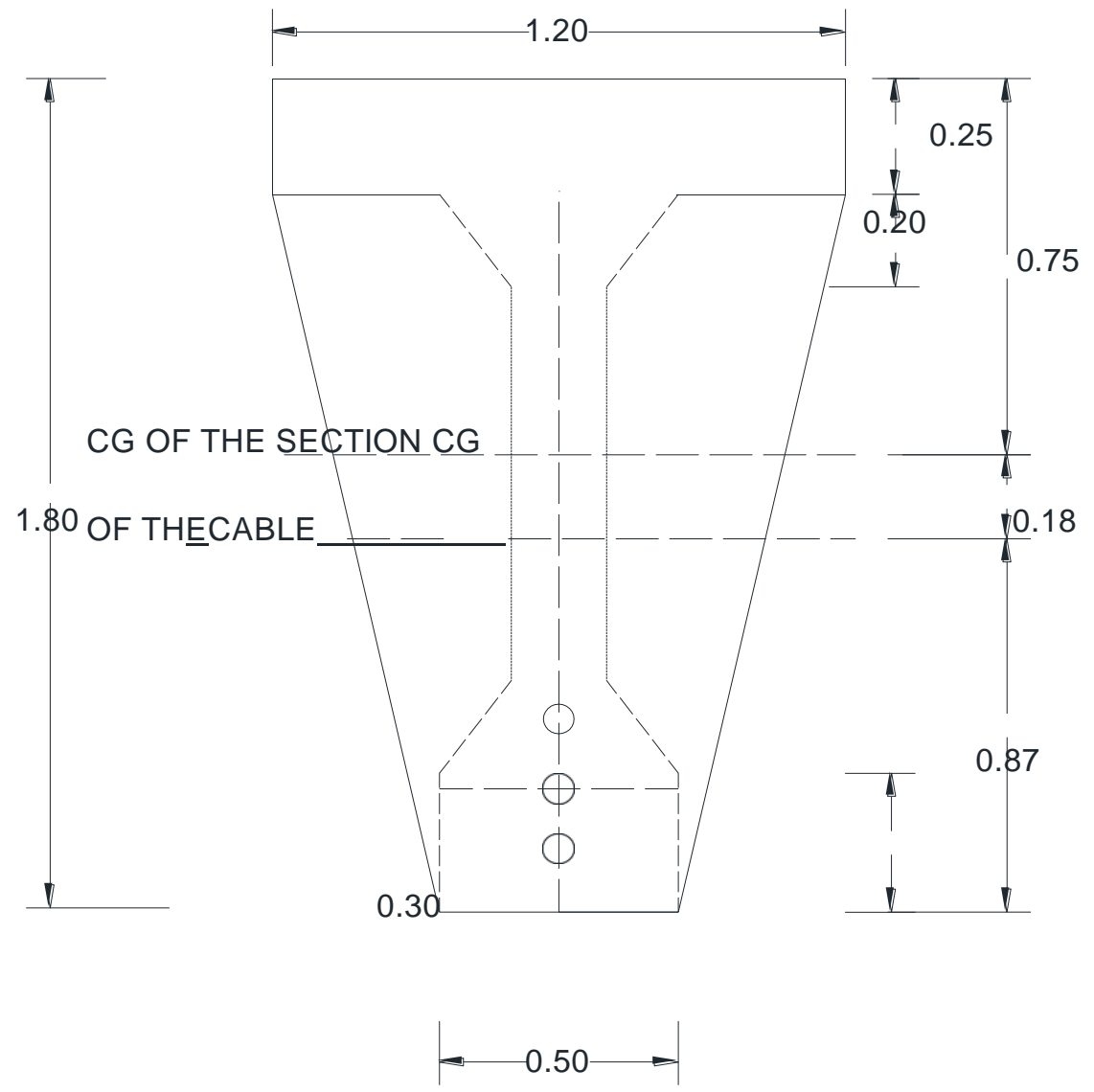

Arrangement of Cables at Support Section 


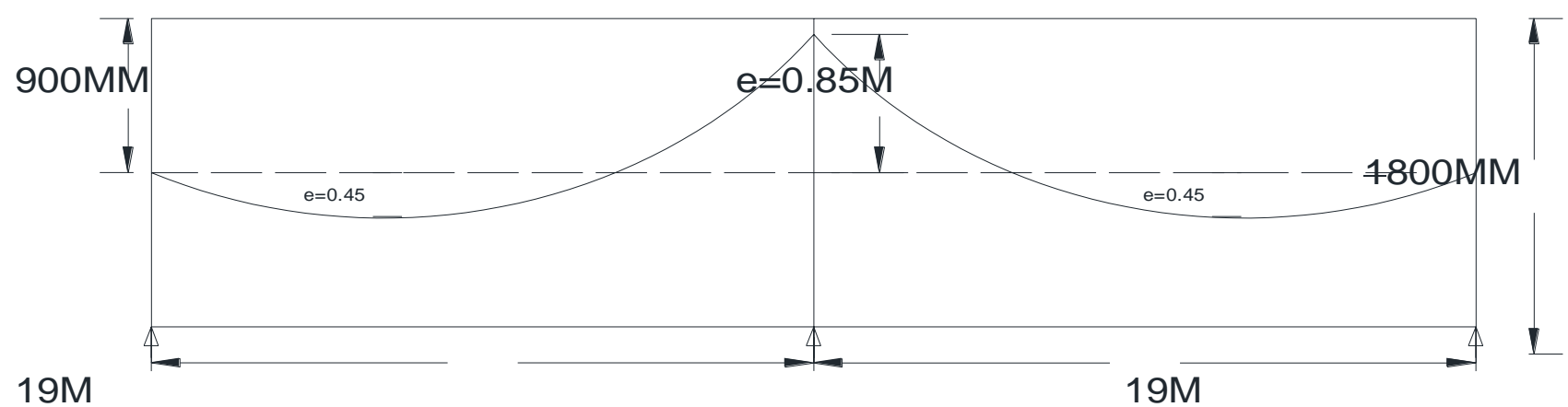

Concordant

Cable Profile

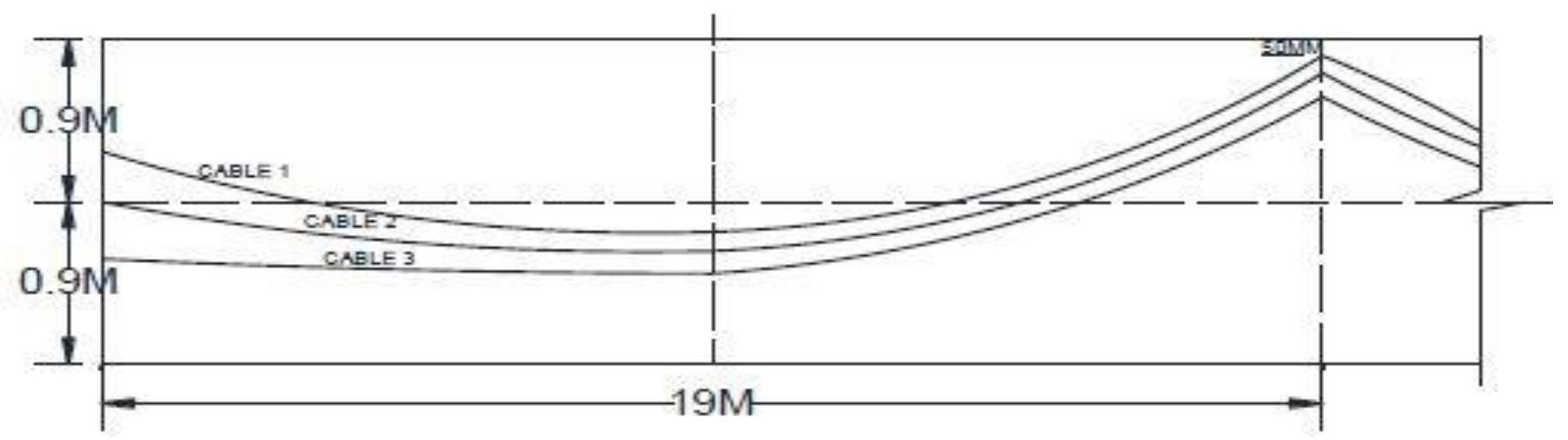

\section{Cable Profile}




\section{Check For Ultimate Flexural Strength:}

For the centre of span section,

Ap $=3 * 7 * 140=2940 \mathrm{~mm}^{2}$

$\mathrm{b}=1200 \mathrm{~mm} ; \quad \mathrm{d}=1600 \mathrm{~mm}$

bw $=200 \mathrm{~mm} ; \quad \mathrm{fck}=50 \mathrm{~N} / \mathrm{mm}^{2} \mathrm{D}_{\mathrm{f}}=250 \mathrm{~mm}$

According to IRC: $18-2000, \mathrm{Mu}=1.5 \mathrm{M}_{\mathrm{G}}+2.5 \mathrm{M}_{\mathrm{L}}$

$=\left(1.5 * 1858.51 * 10^{6}\right)+\left(2.5 * 1437.275^{*} 10^{6}\right)$

$=6380.95 * 10^{6}$

$=6380.95 \mathrm{kN}-\mathrm{m}$

The ultimate flexural strength is computed as,

i) Failure by yielding of steel $M u=0.9 * d * A_{p} * f_{p}$

$=0.9 * 1600 * 2940 * 1862$

$=7882.96 \mathrm{kN}-\mathrm{m}$

ii) Failure by crushing ofmaterials

$\mathrm{Mu}=0.176 \mathrm{~b}_{\mathrm{w}} \mathrm{d}^{2} \mathrm{f}_{\mathrm{ck}}+0.667 * 0.8 *\left(\mathrm{~b}-\mathrm{b}_{\mathrm{w}}\right)\left(\mathrm{d}-\mathrm{D}_{\mathrm{f}} / 2\right) \mathrm{D}_{\mathrm{f}} * \mathrm{f}_{\mathrm{ck}}$

$=(0.176 * 200 * 1600 * 50)+0.667 * 0.8 * 1000 *(1600-(250 / 2)) *(250 * 250)$

$=14343 \mathrm{kN}-\mathrm{m}$

According to IS: 1343-1980, the ultimate flexural strength of the center span section is computed as,

$A p=\left(A_{p w}+A_{p f}\right)$

$\mathrm{A}_{\mathrm{pf}}=0.45 \mathrm{f}_{\mathrm{ck}}\left(\mathrm{b}-\mathrm{b}_{\mathrm{w}}\right)\left(\mathrm{D}_{\mathrm{f}} / \mathrm{f}_{\mathrm{p}}\right)$

$=0.45 * 50 *(1200-200) *(250 / 1862)$

$=3021 \mathrm{~mm}^{2}$

$\therefore$ Apw $=3021-2940$ 
$=81 \mathrm{~mm}^{2}$

Ratio, $\frac{A p w \cdot f p}{b w \cdot d \cdot f c k}=\frac{81 * 1862}{200 * 1400 * 50}$

$=0.0377$

From table 11 of IS: 1343 , we have for post tensioned beams with effective bond, fpu/0.87fp = 1

$\mathrm{fpu}=0.87 * 1862$

$=1619.94$

$\mathrm{X}_{\mathrm{u}} / \mathrm{d}=0.0826$

$\mathrm{X}_{\mathrm{u}}=0.0826^{*} 170 \Theta$

$=115.64 \mathrm{~mm}$

$\therefore \mathrm{Mu}=$ fpu. Ap. $\left(\mathrm{d}-0.42 \mathrm{X}_{\mathrm{u}}\right)$

$=1619.94 * 2940 *(1400-115.64)$

$=6116.92 * 10^{6} \mathrm{kN}-\mathrm{m}$

Check for Ultimate Shear Strength:

Ultimate Shear force $\mathrm{Vu}=(1.5 \mathrm{Vg}+2.5 \mathrm{Vq})$

$=(1.5 * 351.76+2.5 * 494.51)$

$=1763.915 \mathrm{kN}$

According to IRC: 18-2000, the ultimate shear resistance of support section uncracked in flexure is given by, $\mathrm{Vcw}=0.67 \mathrm{bw} \cdot \mathrm{h} \sqrt{ }\left(\mathrm{ft}_{\mathrm{t}}^{2}+0.8 \mathrm{fcp} . \mathrm{f}_{\mathrm{t}}\right)+$ n.P.Sine Where $\mathrm{bw}=$ Width of web $=200 \mathrm{~mm}$ $\mathrm{h}=$ Overall depth of Girder $=1800 \mathrm{~mm}$

$\mathrm{f}_{\mathrm{t}}=$ Maximum principal tensile stress at centroid axis $\mathrm{f}_{\mathrm{t}}=0.24 \sqrt{ } \mathrm{f}_{\mathrm{ck}}=0.24 \sqrt{50}=1.7 \mathrm{~N} / \mathrm{mm}^{2}$

$\mathrm{fcp}=$ Compressive stress at centroidal axis due to prestress

$=\frac{\mathrm{n}^{\mathrm{P}}}{A}$

$=\left(0.8 * 4254 * 10^{3}\right) /\left(0.73 * 10^{6}\right)$ 
$=4.66 \mathrm{~N} / \mathrm{mm}^{2}$

Eccentricity of cables at centre of span $=850 \mathrm{~mm}$ Eccentricity of cables at support $=180 \mathrm{~mm}$

Net eccentricity $=e=(850-180)=670 \mathrm{~mm}$ Slope of the cable $=\Theta=\frac{4 e}{L}$

$=\overline{19 \times 1000}$

$=0.14$

$\because \mathrm{Vcw}=0.67 * 200 * 1800 \sqrt{ }\left(1.7^{2}+0.8 * 4.66 * 1.7\right)+0.8 * 4254810^{3 * 0} 0.14$

$=1209.14 \mathrm{kN}$

Shear resistance required $=1763.915 \mathrm{kN}$ Shear capacity of section $=1209.14 \mathrm{kN}$

Base shear $=(1763.915-1209.14)=554.775 \mathrm{kN}$

Using 10mm diameter 2 legged stirrups of Fe415 HYSD bars, the spacing Sv is obtained as,

$\mathrm{Sv}=\left(0.87 * \mathrm{f}_{\mathrm{y}} * \mathrm{~A}_{\mathrm{sv}} * \mathrm{~d}_{\mathrm{t}}\right) / \mathrm{V}$

$=(0.87 * 415 * 2 * 79 * 1750) /\left(554.775 * 10^{3}\right)$

$=179.94 \mathrm{~mm}$

Provide $10 \mathrm{~mm}$ diameter stirrups at $150 \mathrm{~mm}$ centers near support and gradually increased to $300 \mathrm{~mm}$ towards the center of span.

\section{Supplementary Reinforcement:}

Longitudinal reinforcement of not less than 0.15 percent of gross cross-sectional area to be provided to safeguard against shrinkage cracking.

Ast $=\left[\left(0.15 * 0.73 * 10^{6}\right) / 100\right]$

$=1095 \mathrm{~mm}^{2}$

$20 \mathrm{~mm}$ diameter bars are provided and distributed in the compression flange as shown 


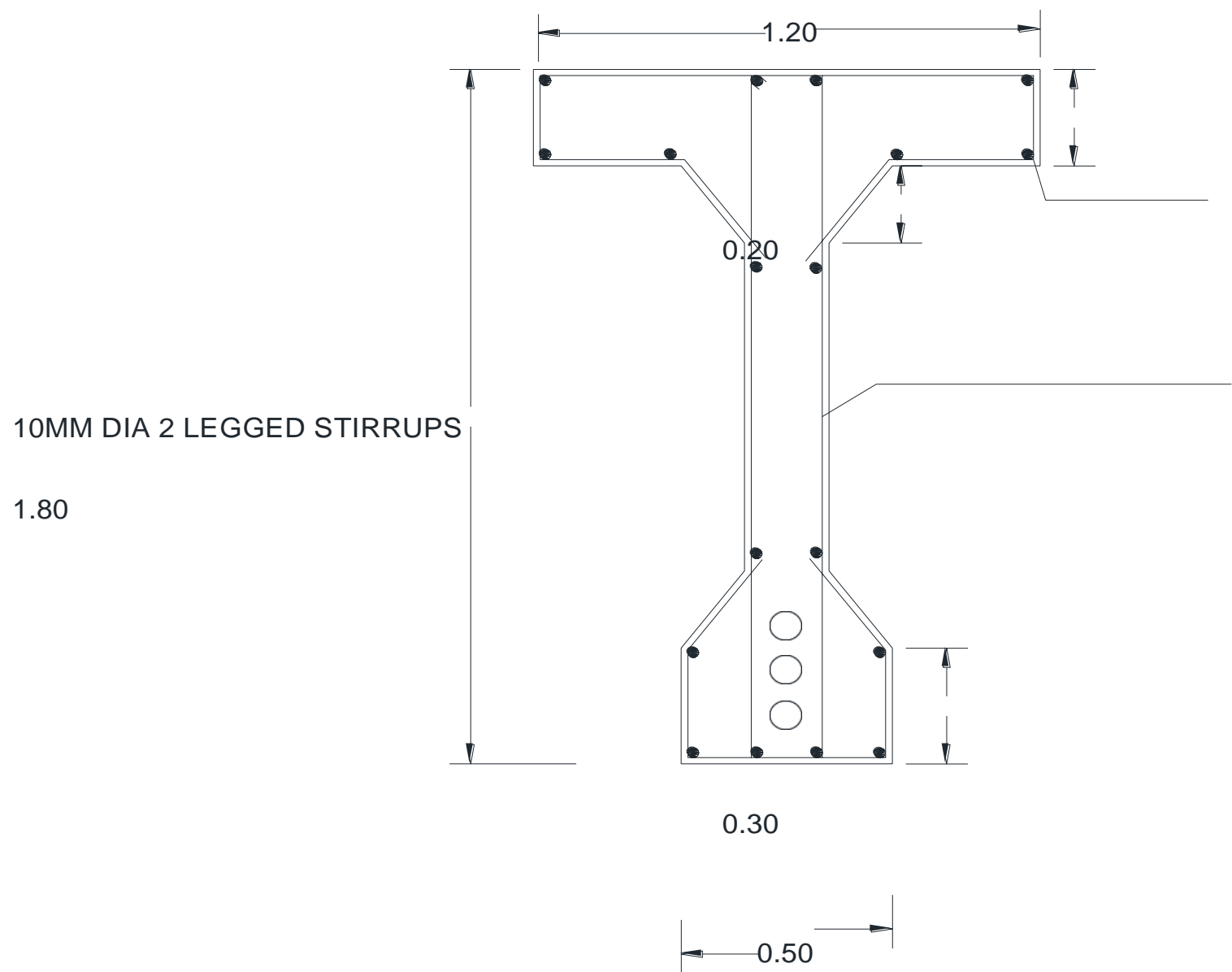




\section{DESIGN OF END BLOCKS:}

Solid end blocks are provided at the end supports over a length of $1.5 \mathrm{~m}$. Typical equivalent prisms on which the anchorage forces are considered to be effective are detailed in the figure. The bursting tension is computed using the data given in the table.

\begin{tabular}{|l|l|l|l|l|l|}
\hline$\left(\mathrm{y}_{\mathrm{po}} / \mathrm{y}_{\mathrm{o}}\right)$ & 0.3 & 0.4 & 0.5 & 0.6 & 0.7 \\
\hline$\left(\mathrm{F}_{\mathrm{bst}} / \mathrm{P}_{\mathrm{k}}\right)$ & 0.23 & 0.20 & 0.17 & 0.14 & 0.11 \\
\hline
\end{tabular}

In the horizontal plane we have the data, $\mathrm{P}_{\mathrm{k}}=1459 \mathrm{kN}$

$2 \mathrm{y}_{\mathrm{po}}=225 \mathrm{~mm} 2 \mathrm{y}_{\mathrm{o}}=900 \mathrm{~mm}$

$\therefore$ The ratio $\left(\mathrm{y}_{\mathrm{po}} / \mathrm{y}_{\mathrm{o}}\right)=(112.5 / 450)=0.25$ Bursting Tension, $\mathrm{F}_{\mathrm{bst}}=(0.23 * 1459)=336 \mathrm{kN}$

Area of stel required to resist this tension is obtained as, As $=\left(336^{*} 10^{3}\right) /(0.87 * 415)=931 \mathrm{~mm}^{2}$

Provide $10 \mathrm{~mm}$ diameter bars at $100 \mathrm{~mm}$ centres in the horizontal direction. In the vertical plane, the ratio of $\left(\mathrm{y}_{\mathrm{po}} / \mathrm{y}_{\mathrm{o}}\right)$ being higher the magnitude of bursting tension is smaller. However the same reinforcements are provided in the form of a mesh both in the horizontal and vertical directions.

\section{INTERMEDIATE SPAN:}

Design of Interior Panel: Bending Moments:

Dead weight of slab $=(1 * 1 * 0.25 * 24)=6.00 \mathrm{kN} / \mathrm{m}^{2}$

Dead weight of wearing coat $=(0.008 * 22)=1.76 \mathrm{kN} / \mathrm{m}^{2}$ Total dead load $=7.76 \mathrm{kN} / \mathrm{m}^{2}$

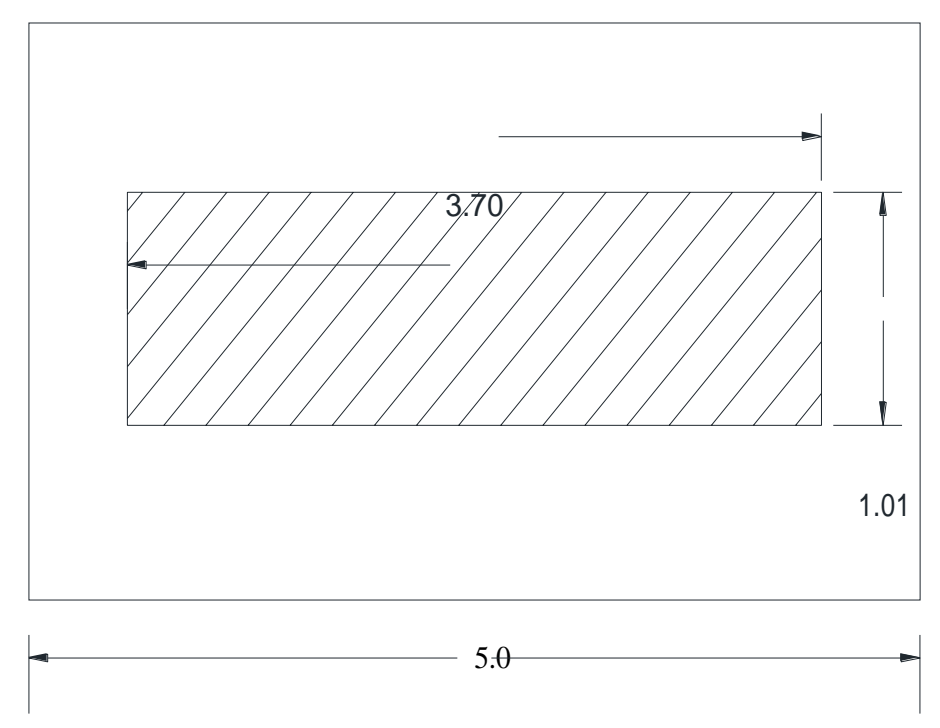


Position of IRC Class AA Wheel Load For Maximum Bending Moment

$\mathrm{U}=\mathrm{B}+2 \mathrm{t} \mathrm{V}=\mathrm{L}+2 \mathrm{t}$

$\mathrm{U}=(0.85+2 * 0.08)=1.01$

$\mathrm{V}=(3.6+2 * 0.08)=3.76$

$\mathrm{u} / \mathrm{B}=1.01 / 2.5=0.404$

$\mathrm{V} / \mathrm{L}=3.76 / 5=0.752$

From Pigeaud ${ }^{\text {ee }}$ s curve, $\mathrm{K}=\mathrm{B} / \mathrm{L}=0.5 \mathrm{~m}_{1}=0.098$ andm $_{2}=0.02$

$\mathrm{M}_{\mathrm{B}}=\mathrm{w}\left(\mathrm{m}_{1}+0.15 \mathrm{~m}_{2}\right)$

$=350\left(0.098+0.15^{*} 0.02\right)$

$=35.35 \mathrm{kN}-\mathrm{m}$

As the slab is continuous, Design $\mathrm{BM}=0.8 \mathrm{MB}$

Design BM including impact and continuity factor

$=1.25 * 0.8 * 35.35$

$=35.35 \mathrm{kN}-\mathrm{m}$

$\mathrm{M}_{\mathrm{L}}=\mathrm{w}\left(\mathrm{m}_{2}+0.15 \mathrm{~m}_{1}\right)$

$=350(0.02+0.15 * 0.098)$

$=12.14 \mathrm{kN}-\mathrm{m}$

Shear Force:

Dispersion in the direction of span,

$=0.85+2(0.08+0.25)$

$=1.51 \mathrm{~m}$

For maximum shear, load is kept such that the whole dispersion is within span. The load is kept at $1.51 / 2=0.755 \mathrm{~m}$ from the edge of the beam.

Effective width of slab $=k x[1-(x / L)]+b_{w}$ Clear length of panel $=4.75-0.2=4.55 \mathrm{~m}$ 
$\therefore \mathrm{B} / \mathrm{L}=4.55 / 2.3=1.978$

From table, (IRC 21-2000) value of $\mathrm{k}$ for continuous slab is $=2.6$ Effective width of slab,

$=2.6 * 0.755[1-(0.755 / 2.3)]+[3.6+(2 * 0.08)]$

$=5.079 \mathrm{~m}$

Load per meter width $=350 / 5.079$

$=70 \mathrm{kN}$

Shear force per meter width $=70(2.3-0.755) / 2.3$

$=47.00 \mathrm{kN}$

Shear force with impact $=1.25 * 47.0=58.75 \mathrm{kN}$

Dead Load BM and Shear Force:

Total dead load $=7.76 \mathrm{kN} / \mathrm{m}^{2}$

Total dead load on panel $=5 * 2.5 * 7.76$

$$
=97 \mathrm{kN}
$$

For $\mathrm{UDL} \mathrm{U} / \mathrm{B}=1$ and $\mathrm{V} / \mathrm{L}=1 \mathrm{~K}=\mathrm{B} / \mathrm{L}=2.5 / 5=0.5$

$1 / \mathrm{K}=1$

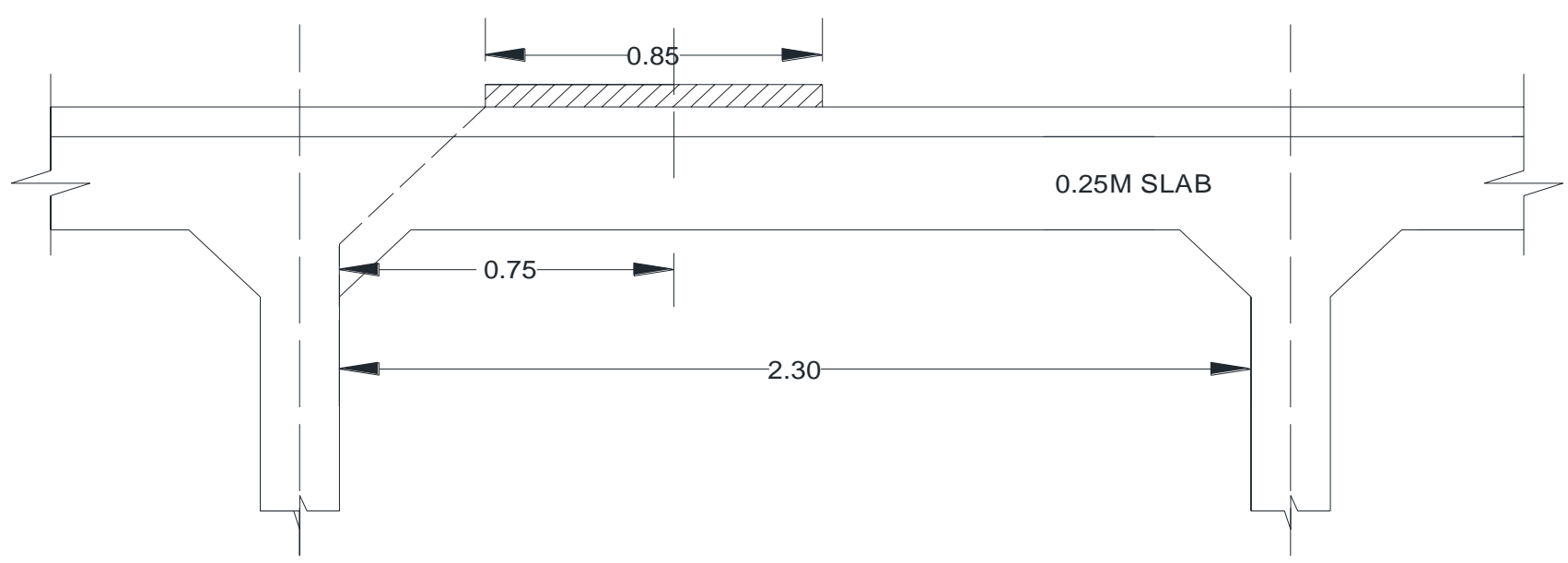

Position Of Wheel Load For Maximum Shear 
From Pigeaud ${ }^{\text {ee }}$ c curve, $\mathrm{m}_{1}=0.047$ and $\mathrm{m}_{2}=0.01$

$\mathrm{M}_{\mathrm{B}}=97\left(0.047+0.15^{*} .01\right)=4.70 \mathrm{kN}-\mathrm{m} \mathrm{M}_{\mathrm{L}}=97(0.01+0.15 * 0.047)=1.65 \mathrm{kN}-\mathrm{m}$

Design $\mathrm{BM}$ including continuity factor, $\mathrm{M}_{\mathrm{B}}=0.8 * 4.7=3.76 \mathrm{kN}-\mathrm{m} \mathrm{M}_{\mathrm{L}}=0.8 * 165=1.32 \mathrm{kN}-\mathrm{m}$

Dead load shear force $=\mathrm{wl} / 2$

$=(7.76 * 2.3) / 2$

$=8.92 \mathrm{kN}$

Design moments and shear forces: Total $\mathrm{M}_{\mathrm{B}}=35.35+3.76=39.11 \mathrm{kN}-\mathrm{m} \mathrm{M}_{\mathrm{L}}=12.14+1.32=13.46 \mathrm{kN}-\mathrm{m}$

Design shear force $=58.75+8.92=67.67 \mathrm{KN}$

\section{Design of Slab Section and Reinforcement:}

Effective depth, $d=\sqrt{ }(\mathrm{M} / \mathrm{Qb})$

$=\sqrt{ }\left(39.11 * 10^{6}\right) /(0.762 * 1000)$

$=226 \mathrm{~mm}$

$d_{\text {provided }}>d_{\text {efective. }}$ Hence ok.

Ast $=\mathrm{M} /\left(\sigma_{\mathrm{st}} * \mathrm{j} * \mathrm{~d}\right)$

$=\left(39.11 * 10^{6}\right) /(200 * 0.96 * 230)$

$=938 \mathrm{~mm}^{2}$

Use $12 \mathrm{~mm}$ dia. Bars @ $120 \mathrm{~mm} \mathrm{c} / \mathrm{c}$ Ast provided $=\left[1000 *\left(* 12^{2}\right) / 4\right] / 120$

$=942.47 \mathrm{~mm}^{2}$

$\pi$

Effective depth for long span using 10mm dia.,

$=230-6-5$

$=219 \mathrm{~mm}$

Ast $=\left(13.46^{*} 10^{6}\right) /(200 * 0.96 * 219)$

$=339 \mathrm{~mm}^{2}$

But minimum reinforcement using HYSD bars according o IRC $18-2000$ is $0.15 \%$ of cross section area. 
Hence,

Ast $=0.0015^{*} 1000 * 250$

$=375 \mathrm{~mm}^{2}$

Check for Shear Stress:

Nominal shear stress $=\tau_{\mathrm{v}}=\mathrm{V} / \mathrm{bd}$

$=\left(58.75 * 10^{3}\right) /(1000 * 230)$

$=0.255 \mathrm{~N} / \mathrm{mm}^{2}$

At support section, Ast $=942 \mathrm{~mm}^{2}$

Hence, $(100$ Ast $) / \mathrm{bd}=(100 * 942) /(1000 * 230)=0.4$ For $\mathrm{M}_{20}$ concrete, $\tau_{\mathrm{c}}=0.25 \mathrm{~N} / \mathrm{mm}^{2}$

(from table 12B IRC 21-2000) For overall depth 250mm, K=1.1 (From table 12C IRC: 21-2000)

$\therefore$ Permissible shear stress in concrete slab $=\mathrm{K} \tau_{\mathrm{c}}$

$=1.10 * 0.25$

$=0.275 \mathrm{~N} / \mathrm{mm}^{2}>\tau_{\mathrm{v}=} 0.19 \mathrm{~N} / \mathrm{mm}^{2}$ Hence the shear stresses are within the safe permissible limits.

\section{Design of Longitudinal Girders:}

\section{Reaction Factors:}

Using Courbon"es theory, the IRC class AA loads are arranged for maximum eccentricity as shown in fig.

Reaction factor for outer girder $\mathrm{A}$ is, $\mathrm{R}_{\mathrm{A}}=\left(\Sigma \mathrm{w}_{1} / \mathrm{n}\right) *\left[1+\left(\operatorname{nex}_{1}\right) /\left(\Sigma \mathrm{x}^{2}\right)\right]$

$=\left(2 \mathrm{w}_{1} / 4\right) *\left[1+(4 * 1.1 * 3.75) /\left\{\left(2 * 3.75^{2}\right)+\left(2 * 1.25^{2}\right)\right\}\right]=\underline{0.764} \mathrm{w}_{1}$ 


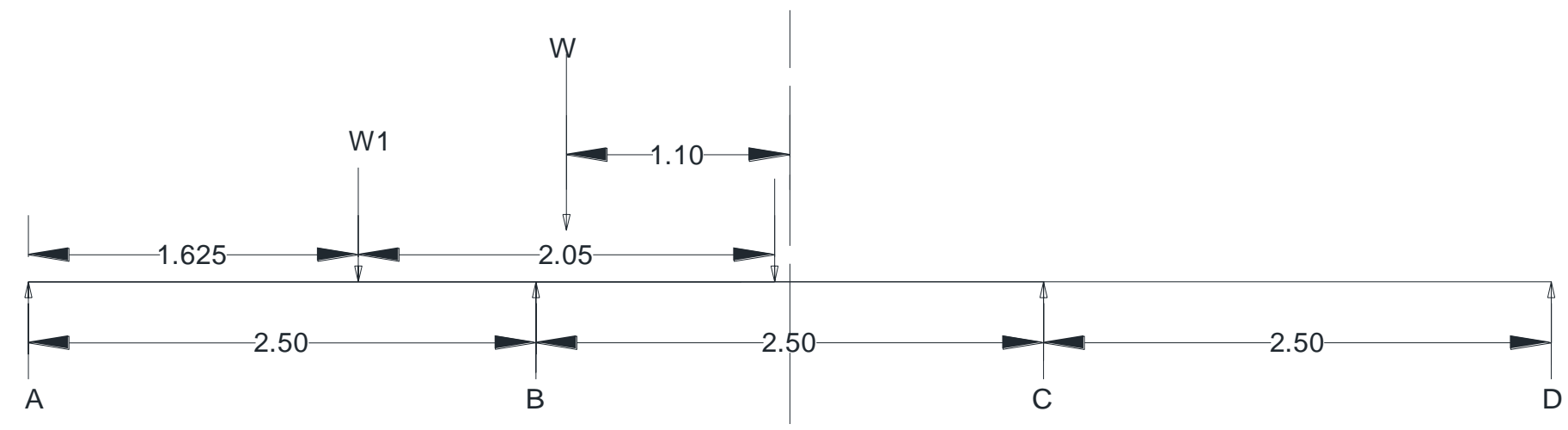

\section{Transverse Disposition of IRC Class AA Tracked Vehicle}

Reaction factor for inner girder B is ,

$\left.\mathrm{R}_{\mathrm{B}}=2 \mathrm{w}_{1} / 4\right) *\left[1+\left(4 * 1.25^{*} 1.1\right) /\left\{\left(2 * 3.75^{2}\right)+\left(2 * 1.25^{2}\right)\right\}\right]$

$=0.588 \mathrm{w}_{1}$

If $\mathrm{w}_{1}=0.5 \mathrm{w} \mathrm{R}_{\mathrm{A}}=0.764 * 0.5 \mathrm{w}$

$=0.382 \mathrm{w}$

$\mathrm{R}_{\mathrm{B}}=0.588 * 0.5 \mathrm{w}$

$=0.294 \mathrm{w}$ 
Dead Load From Slab Per Girder:

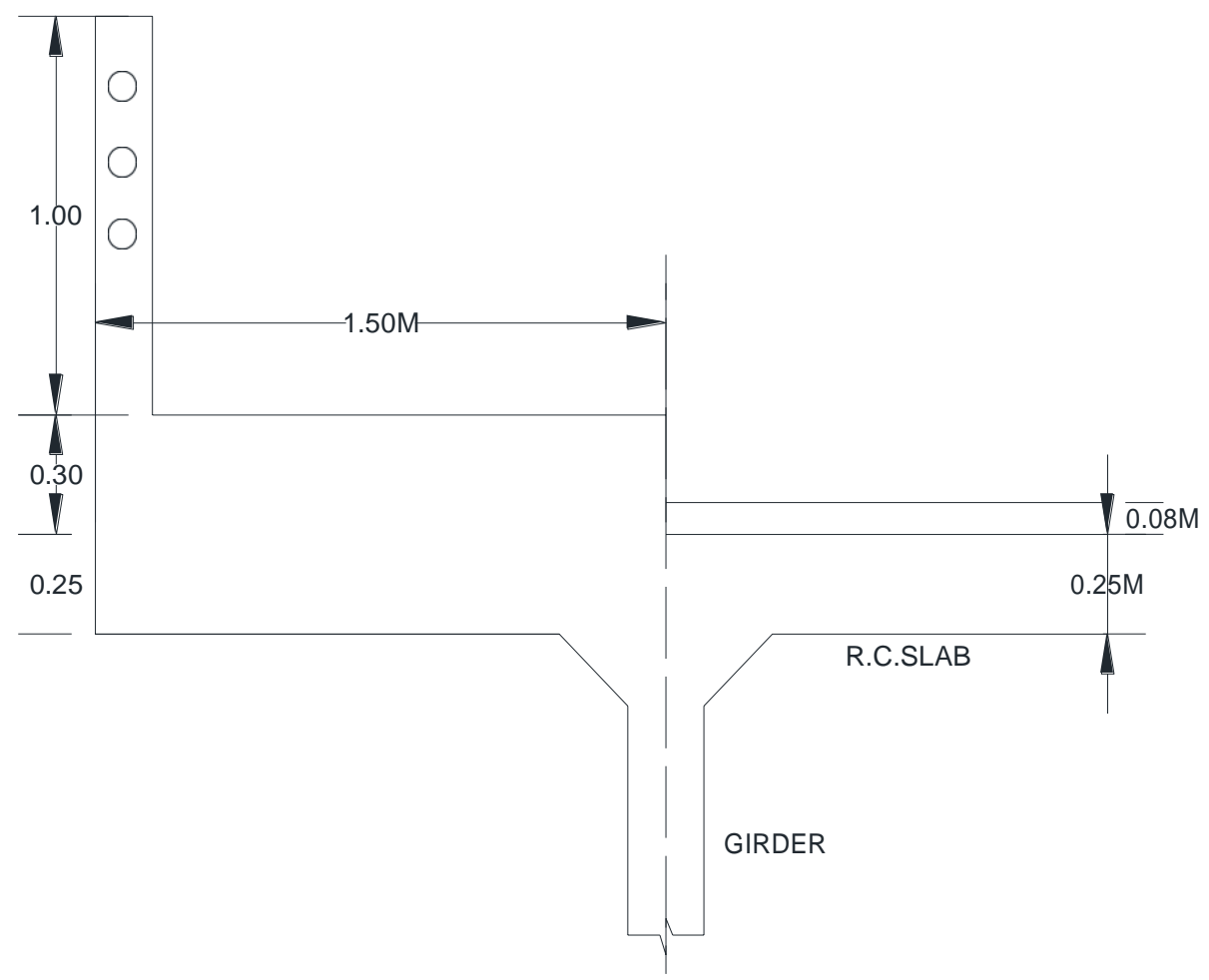

Weight of ,

Parapet railing (lump sum) $=0.92 \mathrm{kN} / \mathrm{m}$

Foot path and kerb $=0.3 * 1.5 * 24=10.08 \mathrm{kN} / \mathrm{m}$ Deck slab $=0.25 * 1.5 * 24=9 \mathrm{kN} / \mathrm{m}$

Total load on deck slab $=[(2 * 20)+(7.76 * 7.5)]=98.20 \mathrm{kN} / \mathrm{m}$

It is assumed that the deck load is shared equally by all the four girders.

$\therefore$ Dead load per girder $=98.20 / 4=24.55 \mathrm{kN} / \mathrm{m}$

Dead Load On Main Girder:

Overall depth of girder $=1800 \mathrm{~mm}$

Dead weight of $\mathrm{rib}=1.15 * 0.3 * 24=8.28 \mathrm{kN} / \mathrm{m}$

Dead weight of bottom flange $=\left(0.5^{*} 0.4 * 24\right)=4.80 \mathrm{kN} / \mathrm{m}$ Totalload $\quad=13.08 \mathrm{kN} / \mathrm{m}$ 
Weight of cross girder $=(0.2 * 1.25 * 24)=6 \mathrm{kN} / \mathrm{m}$

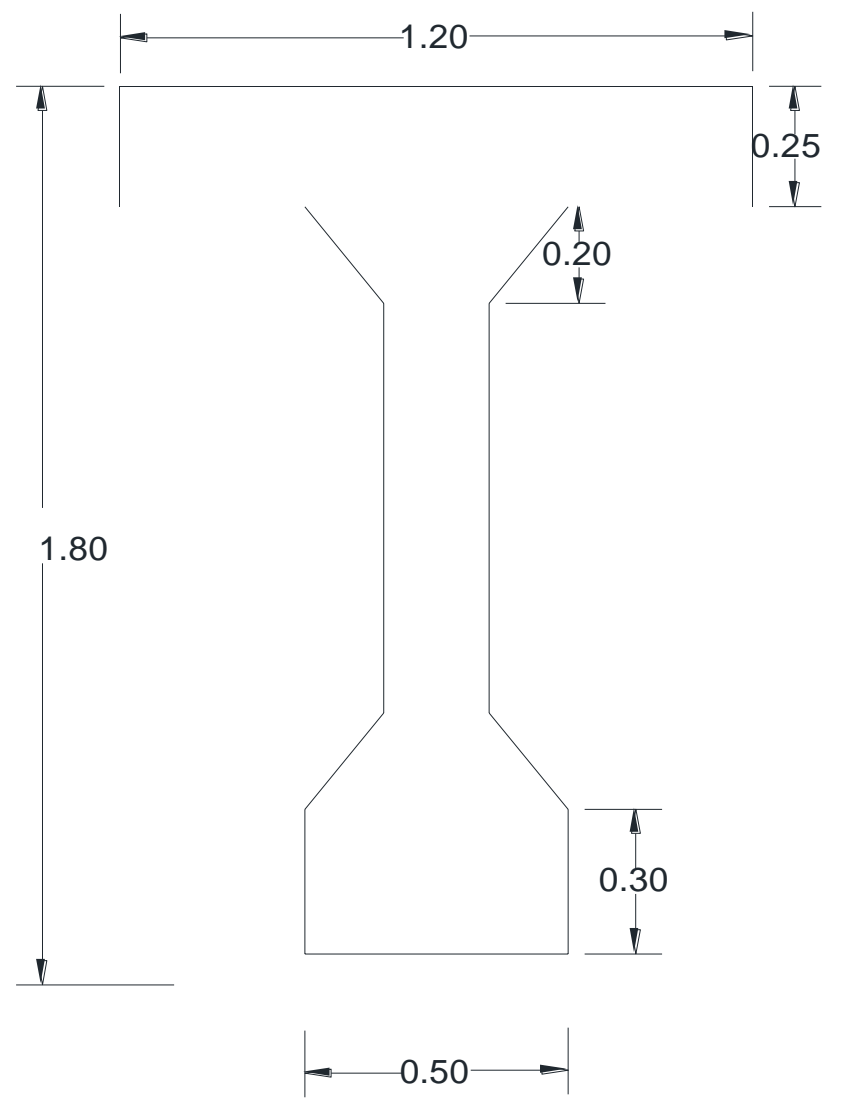

Cross section of Prestressed Concrete Girder Dead Load

Moment and Shear In Main Girder:

Reaction from deck slab on each girder $=24.55 \mathrm{kN} / \mathrm{m}$ Weight of cross girder $=6 \mathrm{kN} / \mathrm{m}$

Reaction on main girder $=\left(6^{*} 2.5\right)=15 \mathrm{kN} / \mathrm{m}$ Self-weight of main girder $=13.08 \mathrm{kN} / \mathrm{m}$

Total dead load on girder $=(24.55+13.08)=37.63 \mathrm{kN} / \mathrm{m}$

The maximum dead load bending moment and shear force is computed using the loads shown in fig.

$\operatorname{Mmax}=\left[\left(0.125 * 34.87 * 19^{2}\right)+(0.25 * 15 * 19)+(15 * 9.5)+(15 * 4.75)\right.$

$=1858.51 \mathrm{kN}-\mathrm{m}$ 


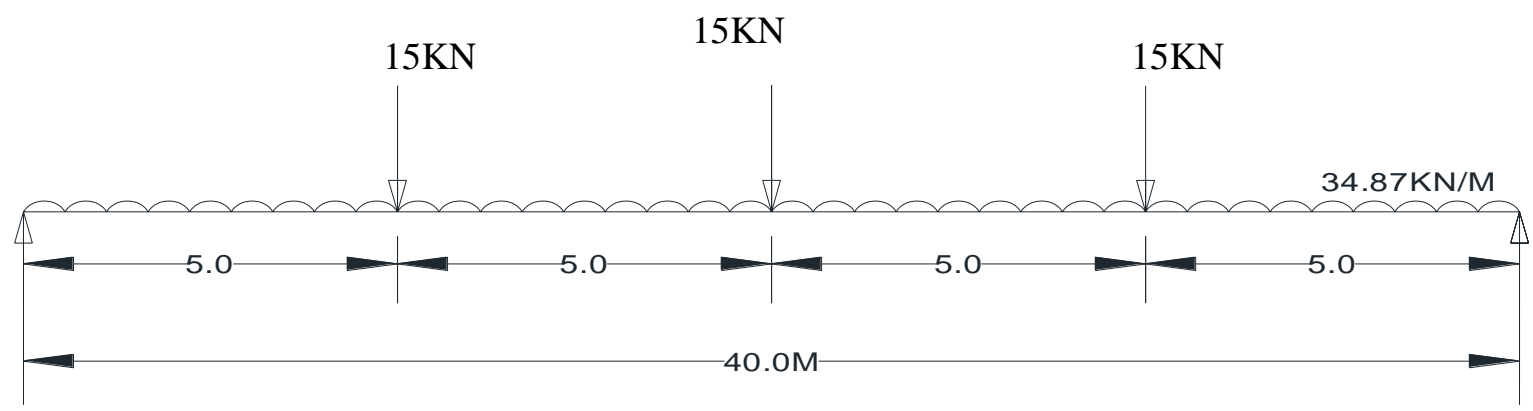

\section{Dead Load on Main Girder}

Dead load shear at support

$V \max =[(0.5 * 34.87 * 19)+(0.5 * 15 * 3)$

$=\underline{\underline{353.76 \mathrm{kN}}}$

Live Load Bending Moment In Girder:

Span of the girder $=40 \mathrm{~m}$

Impact factor $($ class AA $)=10 \%$

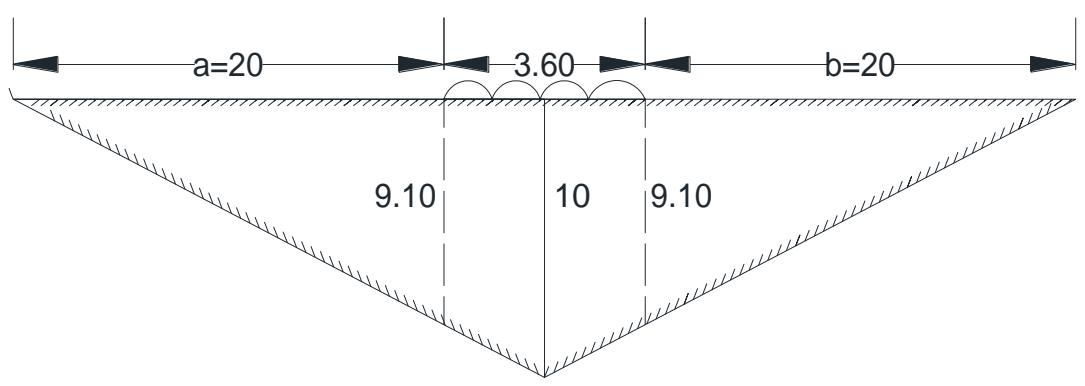

Influence line diagram for Bending Moment in Girder

Live load is placed centrally on the span Bending moment at centre of span

$=0.5 *(9.1+10) * 700$

$=6685 \mathrm{kN}-\mathrm{m}$ 
Bending moment including impact and reaction factor for outer girder is, Live load bending moment $=$ $6685 * 1.1 * 0.382=2809.04 \mathrm{kN}-\mathrm{m}$

For inner girder, $\mathrm{BM}=6685 * 1.1 * 0.294=2161.93 \mathrm{kN}-\mathrm{m}$

\section{Live Load Shear Forces In Girder:}

For maximum live load shear in the girder, the IRC class AA loads are placed as, Reaction of $\mathrm{w}_{2}$ on girder B $=(350 * 0.45) / 2.5=63 \mathrm{kN}$

Reaction of $\mathrm{w}_{2}$ on girder $\mathrm{A}=(350 * 2.05) / 2.5=287 \mathrm{kN}$ Total load on girder $\mathrm{B}=350+63=413 \mathrm{kN}$

Maximum reaction in girder $\mathrm{B}=(413 * 38.2) / 40=394.41 \mathrm{kN}$

Maximum reaction in girder $\mathrm{A}=(287 * 38.2) / 40=274.08 \mathrm{kN}$

Maximu live load shear with impact factor in inner girder $=394.41 * 1.1=433.95 \mathrm{kN}$

Outer girder $=274.08 * 1.1=301.488 \mathrm{kN}$

Design Bending Moment and Shear Force:

\begin{tabular}{|c|c|c|c|c|}
\hline $\begin{array}{l}\text { Bending } \\
\text { Moment }\end{array}$ & $\begin{array}{c}\text { Dead Load } \\
\text { BM }\end{array}$ & Live load BM & Total BM & Unit \\
\hline Outer girder & 8126 & 2809.04 & 10935.04 & $\mathrm{kN}-\mathrm{m}$ \\
\hline Inner girder & 8126 & 2161.93 & 10287.93 & $\mathrm{kN}-\mathrm{m}$ \\
\hline Shear force & Dead load SF & Live load SF & Total SF & Unit \\
\hline Outer girder & 805.1 & 301.48 & 1106.58 & $\mathrm{kN}$ \\
\hline Inner girder & 805.1 & 433.95 & 1239.05 & $\mathrm{kN}$ \\
\hline
\end{tabular}




\section{PROPERTIES OF MAIN GIRDER SECTION:}

Cross sectional area $=(1200 * 250)+(1150 * 300)+(400 * 500)=8.45 * 10^{5} \mathrm{~mm}^{2}$

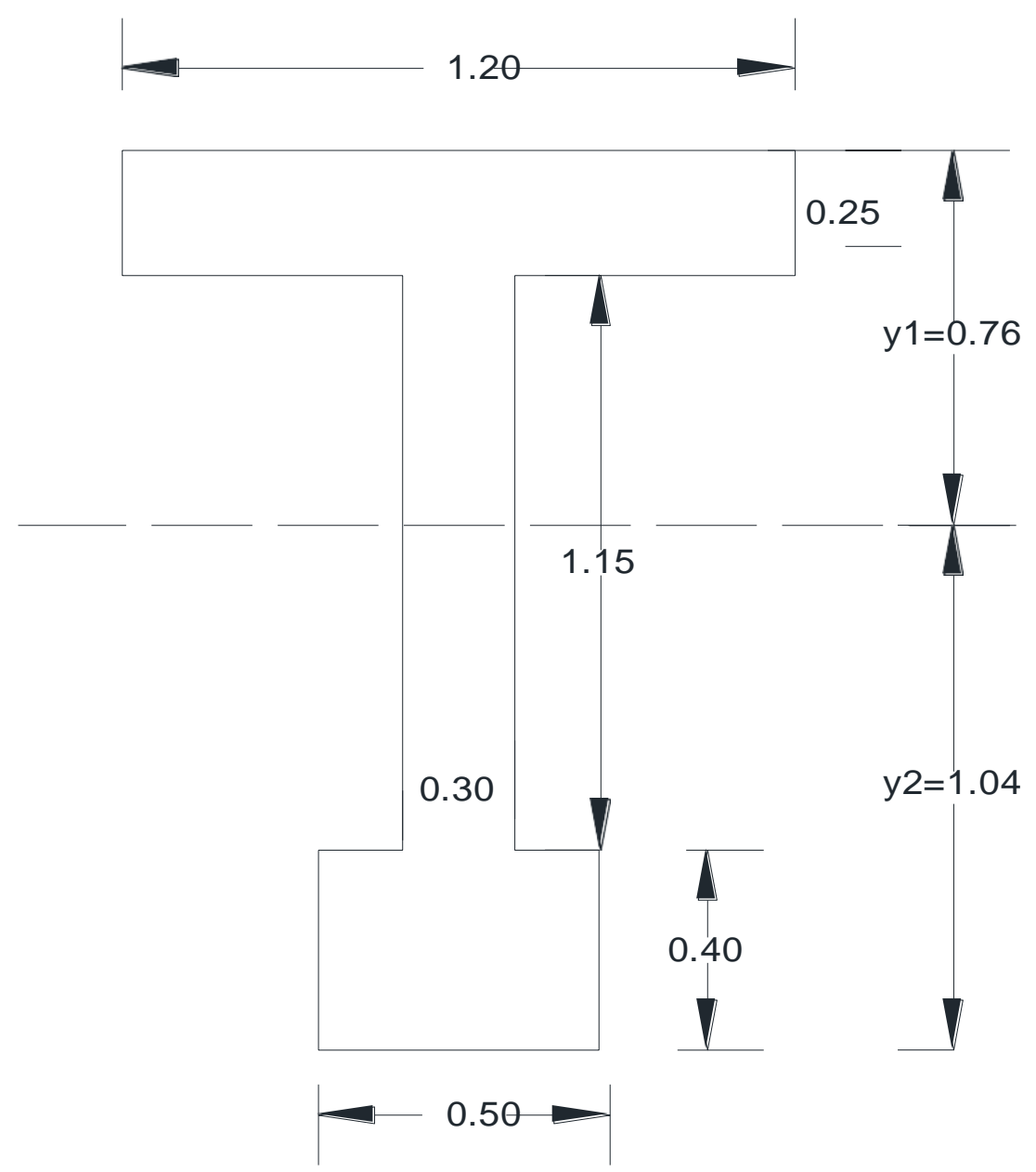

Cross- Section of Main Girder

To locate position of centroid, $\mathrm{Y}_{1}=\Sigma \mathrm{ay}^{1} / \Sigma \mathrm{a}$

$=759.91 \mathrm{~mm}=760 \mathrm{~mm}$

$$
=\frac{(1200 * 250) 125+(1150 * 300) 825+(500 * 400) 1600}{(1200 * 250)+(1150 * 300)+(500 * 400)}
$$

$\mathrm{Y}_{2}=1800-760=1040 \mathrm{~mm}$ 
Moment of inertia about axis of bending,

$\mathrm{I}=\left[\left\{\left(1200 * 250^{3}\right) / 12\right\}+(1200 * 250)(760-125)^{2}\right]+\left[\left\{\left(1150 * 300^{3}\right) / 12\right\}+(1150 * 300)(760-825)^{2}\right]+$ $\left[\left\{\left(500 * 400^{3}\right) / 12\right\}+(500 * 400)(760-1600)^{2}\right]$

$=3.057 * 10^{11} \mathrm{~mm}^{4}$

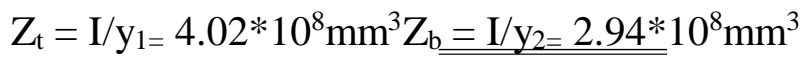

Check for Minimum Section Modulus:

$\mathrm{f}_{\mathrm{ck}}=50 \mathrm{~N} / \mathrm{mm}^{2}$

$\mathrm{f}_{\mathrm{ci}}=40 \mathrm{~N} / \mathrm{mm}^{2}$;

$\mathrm{M}_{\mathrm{L}}=2809.04 \mathrm{kN}-\mathrm{m}$;

$F_{b r}=\eta f_{c t}-f_{t w}$

$=0.80 * 18-0$

$=15.30 \mathrm{~N} / \mathrm{mm}^{2}$

$F_{t r}=f_{c w}-\eta f_{t t}$

$=16 \mathrm{~N} / \mathrm{mm}^{2}$

Inferior stress $=f_{\text {inf }}=\left(f_{t w} / \eta\right)+\left(M_{D} / \eta Z_{b}\right)$

$=0+\left(10935.04 * 10^{6}\right) /\left(0.80 * 2.94 * 10^{8}\right)$

$=43.75 \mathrm{~N} / \mathrm{mm}^{2}$

Minimum section modulus required, $\mathrm{Z}_{\mathrm{required}}=\left[\mathrm{M}_{\mathrm{L}}+(1-\eta) \mathrm{M}_{\mathrm{G}}\right] / \mathrm{F}_{\mathrm{br}}$

$=\left[2809.04 * 10^{6}+(1-0.80) 8126 * 10^{6}\right] / 15.30$

$$
=2.63 * 10^{8} \mathrm{~mm}^{3}<2.94 * 10^{8} \mathrm{~mm}^{3}
$$

Hence the section provided is adequate. 


\section{Pre-Stressing Force:}

Assume a cover of $200 \mathrm{~mm}$

Maximum possible eccentricity e $=(1040.08-200)=840.08 \mathrm{~mm}$ Prestressing force is obtained as,

$\mathrm{P}=\left(\right.$ A. $\left.\mathrm{finf}_{\mathrm{in}} \mathrm{Z}_{\mathrm{b}}\right) /\left(\mathrm{Z}_{\mathrm{b}}+\mathrm{A} . \mathrm{e}\right)$

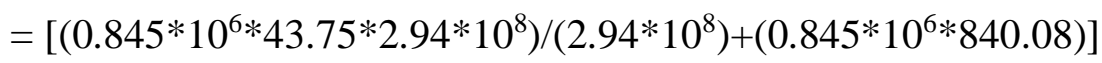

$=10826.94 * 10^{3} \mathrm{~N}$

$=10826.94 \mathrm{kN}$

Using Freyssinet system, anchorage type 7K-15 ( 7 strands of $15.2 \mathrm{~mm}$ diameter ) in $65 \mathrm{~mm}$ cab les duct, (IS:6006-1983)

Force in each cable $=(7 * 0.8 * 280.7)=1459 \mathrm{kN}$ No. of cables $=10826.94 / 1459=7.41=8$ no.s Area of each strand $=140 \mathrm{~mm}^{2}$

Area of 7 strands in each canle $=7 * 140=980 \mathrm{~mm}^{2}$

Area of each strand in 3 cables $=A_{p}=8 * 980=7840 \mathrm{~mm}^{2}$ The cables are arranged at centre of span asshown

\section{Permissible Tendon Zone:}

At Support section, $\mathrm{e}=<\left(\mathrm{Z}_{\mathrm{b}} \cdot \mathrm{f}_{\mathrm{ct} / \mathrm{P}} \mathrm{P}\right)-\left(\mathrm{Z}_{\mathrm{b}} / \mathrm{A}\right)$

$=<\left[\left(2.94 * 10^{8 * 18)} /\left(10826.94 * 10^{3}\right)\right]-\left(2.94 * 10^{8}\right) /\left(0.845^{*} 10^{6}\right)\right.$

$=<140.85 \mathrm{~mm}$

And $\mathrm{e}=>\left(\mathrm{Z}_{\mathrm{b}} \cdot \mathrm{f}_{\mathrm{tw} / \mathrm{r}} \mathrm{P}\right)-\left(\mathrm{Z}_{\mathrm{b}} / \mathrm{A}\right)$

$=>0-\left(2.94 * 10^{8}\right) /\left(0.845^{*} 10^{6}\right)$

$=>-347.928 \mathrm{~mm}$ 


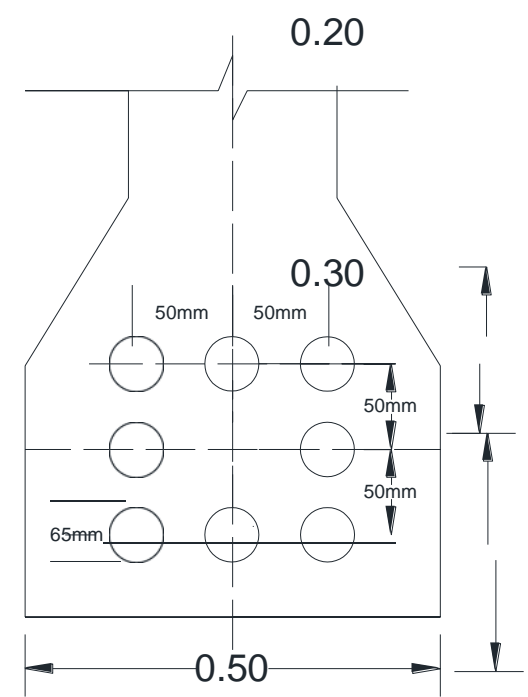

\section{Arrangement of Cable at Centre of Span section Check for Stresses:}

For the centre of the span section, we have $\mathrm{P}=10826.94 \mathrm{kN}$

$\mathrm{e}=840 \mathrm{~mm}$

$\mathrm{A}=0.845^{*} 10^{6} \mathrm{~mm}^{2}$

$\mathrm{Z}_{\mathrm{t}}=4.02 * 10^{8} \mathrm{~mm}^{3}$

$\mathrm{Z}_{\mathrm{b}}=2.9 * 10^{8} \mathrm{~mm}^{3}$

$\mathrm{M}_{\mathrm{L}}=2809.04 \mathrm{kN}-\mathrm{m}$

$\mathrm{M}_{\mathrm{G}}=8126 \mathrm{kN}-\mathrm{m}$

At transfer stage,

$\sigma_{\mathrm{t}}=\left[(\mathrm{P} / \mathrm{A})-\left(\mathrm{Pe} / \mathrm{Z}_{\mathrm{t}}\right)+\left(\mathrm{M}_{\mathrm{L}} / \mathrm{Z}_{\mathrm{t}}\right)\right]$

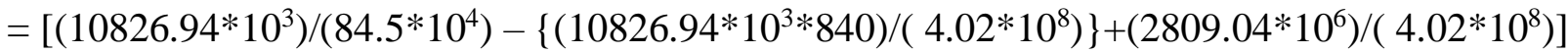

$=12.81-22.62+20.21$

$=10.4 \mathrm{~N} / \mathrm{mm}^{2}$

$\sigma_{\mathrm{b}}=\left[(\mathrm{P} / \mathrm{A})+\left(\mathrm{Pe} / \mathrm{Z}_{\mathrm{b}}\right)-\left(\mathrm{M}_{\mathrm{L}} / \mathrm{Z}_{\mathrm{b}}\right)\right]$

$=\left[\left(10826.94 * 10^{3}\right) /\left(84.5 * 10^{4}\right)+\left\{\left(10826.94 * 10^{3} * 840\right) /\left(2.9 * 10^{8}\right)\right\}-\left(2809.04 * 10^{6}\right) /\left(2.9 * 10^{8}\right)\right]$

$=12.81+30.93-27.63$

$=16.11 \mathrm{~N} / \mathrm{mm}^{2}$ 
At working load stage,

$\sigma_{\mathrm{t}}=\left[(\mathrm{nP} / \mathrm{A})-\left(\mathrm{nPe} / \mathrm{Z}_{\mathrm{b}}\right)+\left(\mathrm{M}_{\mathrm{D}} / \mathrm{Z}_{\mathrm{t}}\right)\right]$

$=\left[\left(0.85 * 10826.94 * 10^{3}\right) /\left(84.5^{*} 10^{4}\right)-\left\{\left(0.85 * 10826.94 * 10^{3 * 840)} /\left(2.9 * 10^{8}\right)\right\}+\left(8126^{*} 10^{6}\right) /\left(4.02 * 10^{8}\right)\right]\right.$

$=18.85 \mathrm{~N} / \mathrm{mm}^{2}$

$\sigma_{\mathrm{b}}=\left[(\eta \mathrm{P} / \mathrm{A})+\left(\eta \mathrm{Pe} / \mathrm{Z}_{\mathrm{t}}\right)-\left(\mathrm{M}_{\mathrm{D}} / \mathrm{Z}_{\mathrm{b}}\right)\right]$

$=\left[\left(0.85^{*} 10826.94 * 10^{3}\right) /\left(84.5 * 10^{4}\right)-\left\{\left(0.85^{*} 10826.94 * 10^{3 * 840)}\right) /\left(4.02 * 10^{8}\right)\right\}+\left(8126^{*} 10^{6}\right) /\left(2.9 * 10^{8 /}\right)\right]$

$=0.129 \mathrm{~N} / \mathrm{mm}^{2}$
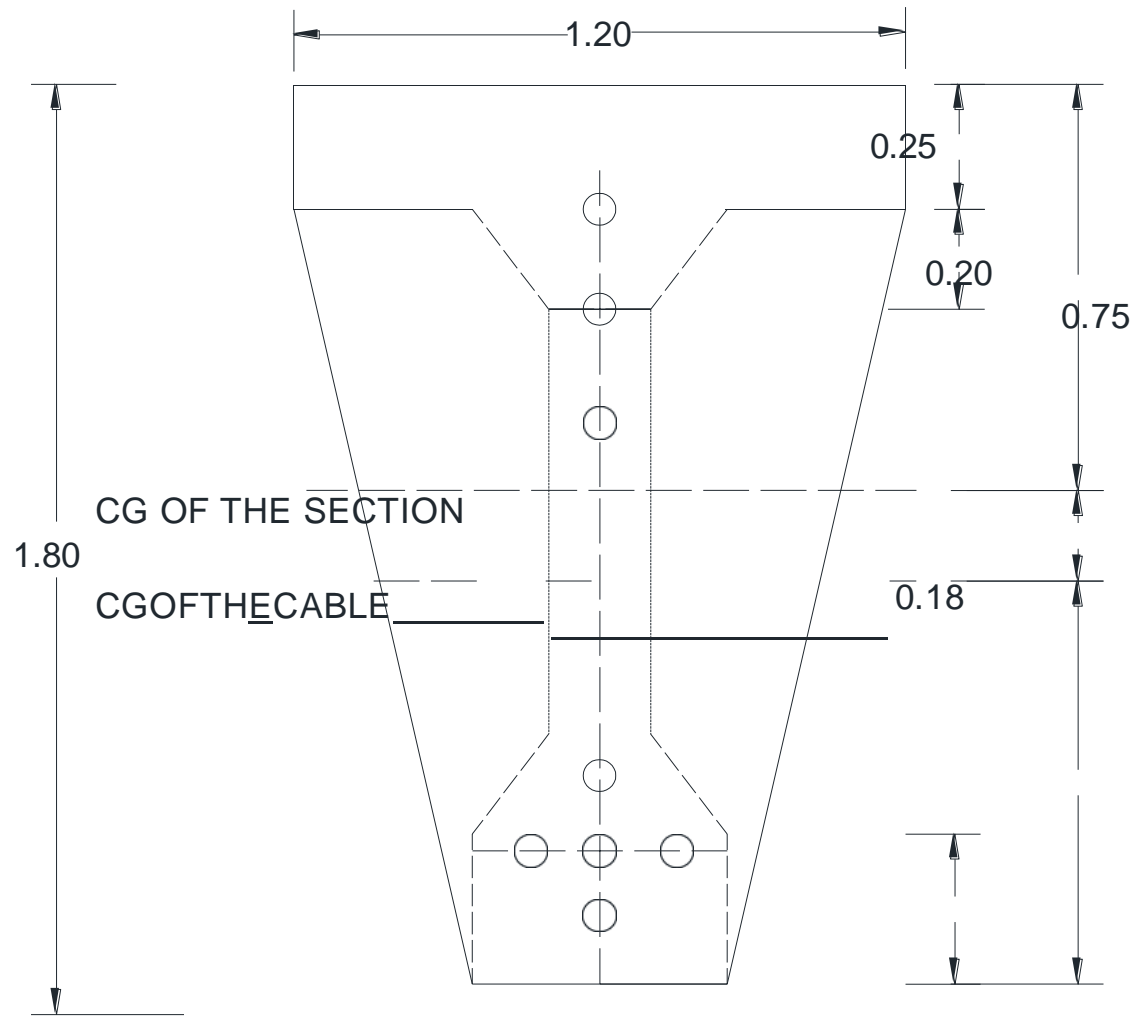


\section{Check for Ultimate Flexural Strength:}

For the center of span section,

$\mathrm{Ap}=8 * 7 * 140=7840 \mathrm{~mm}^{2}$

$\mathrm{b}=1200 \mathrm{~mm} ; \quad \mathrm{d}=1600 \mathrm{~mm}$

bw $=300 \mathrm{~mm} ; \quad$ fck $=50 \mathrm{~N} / \mathrm{mm}^{2} \mathrm{D}_{\mathrm{f}}=250 \mathrm{~mm}$

According to IRC: $18-2000, \mathrm{Mu}=1.5 \mathrm{M}_{\mathrm{G}}+2.5 \mathrm{M}_{\mathrm{L}}$

$=\left(1.5 * 8126 * 10^{6}\right)+\left(2.5 * 2809.04 * 10^{6}\right)$

$=19211.60 * 10^{6}$

$\equiv 19211.60 \mathrm{kN}-\mathrm{m}$

The ultimate flexural strength is computed as,

iii) Failure by yielding of steel $\mathrm{Mu}=0.9 * d * A_{p} * f_{p}$

$=0.9 * 1600 * 7840 * 1862$

$=21021 \mathrm{kN}-\mathrm{m}$

iv) Failure by crushing ofmaterials

$\mathrm{Mu}=0.176 \mathrm{~b}_{\mathrm{w}} \mathrm{d}^{2} \mathrm{f}_{\mathrm{ck}}+0.667 * 0.8 *\left(\mathrm{~b}-\mathrm{b}_{\mathrm{w}}\right)\left(\mathrm{d}-\mathrm{D}_{\mathrm{f}} / 2\right) \mathrm{D}_{\mathrm{f}} * \mathrm{f}_{\mathrm{ck}}$

$=\left(0.176 * 300 * 1600^{2} * 50\right)+0.667 * 0.8 * 900 *(1600-(250 / 2)) *(250 * 250)$

$=51008 \mathrm{kN}-\mathrm{m}$

According to IS: 1343-1980, the ultimate flexural strength of the centre span section is computed as,

$\mathrm{Ap}=\left(\mathrm{A}_{\mathrm{pw}}+\mathrm{A}_{\mathrm{pf}}\right)$

$A_{p f}=0.45 f_{c k}\left(b-b_{w}\right)\left(D_{f} / f_{p}\right)$

$=0.45 * 50 *(1200-300) *(250 / 1862)$

$=2718.85 \mathrm{~mm}^{2}$

$\therefore$ Apw $=7840-2718.85$

$=5121.15 \mathrm{~mm}^{2}$

Ratio, $\frac{A p w_{\cdot f p}}{b w \cdot d \cdot f c k}=\frac{5121.85 * 1862}{300 * 1600 * 50}$ 
$=0.397$

From table 11 of IS: 1343 , we have for post tensioned beams with effective bond, $\mathrm{pu} / 0.87 \mathrm{fp}=0.9$ $\mathrm{fpu}=0.87 * 0.9 * 1862$

$$
\begin{gathered}
=1457.95 \\
X_{u} / d=0.653
\end{gathered}
$$

$X_{u}=0.653 * 1600=$

$=1044.8 \mathrm{~mm}$

$\therefore \mathrm{Mu}=$ fpu. Apw. $\left(\mathrm{d}-0.42 \mathrm{X}_{\mathrm{u}}\right)+0.45 \mathrm{fck}(\mathrm{b}-\mathrm{bw}) \mathrm{Df}(\mathrm{d}-0.5 \mathrm{Df})$

$=[1457.95 * 5121.15 *(1600-0.42 * 1044.8)]+[0.45 * 50 * 900 * 250 *(1600-0.5 * 250)$

$=16134 * 10^{6} \mathrm{kN}-\mathrm{m}$

Check for Ultimate Shear Strength:

Ultimate Shear force $\mathrm{Vu}=(1.5 \mathrm{Vg}+2.5 \mathrm{Vq})$

$=(1.5 * 805.1+2.5 * 433.95)$ 


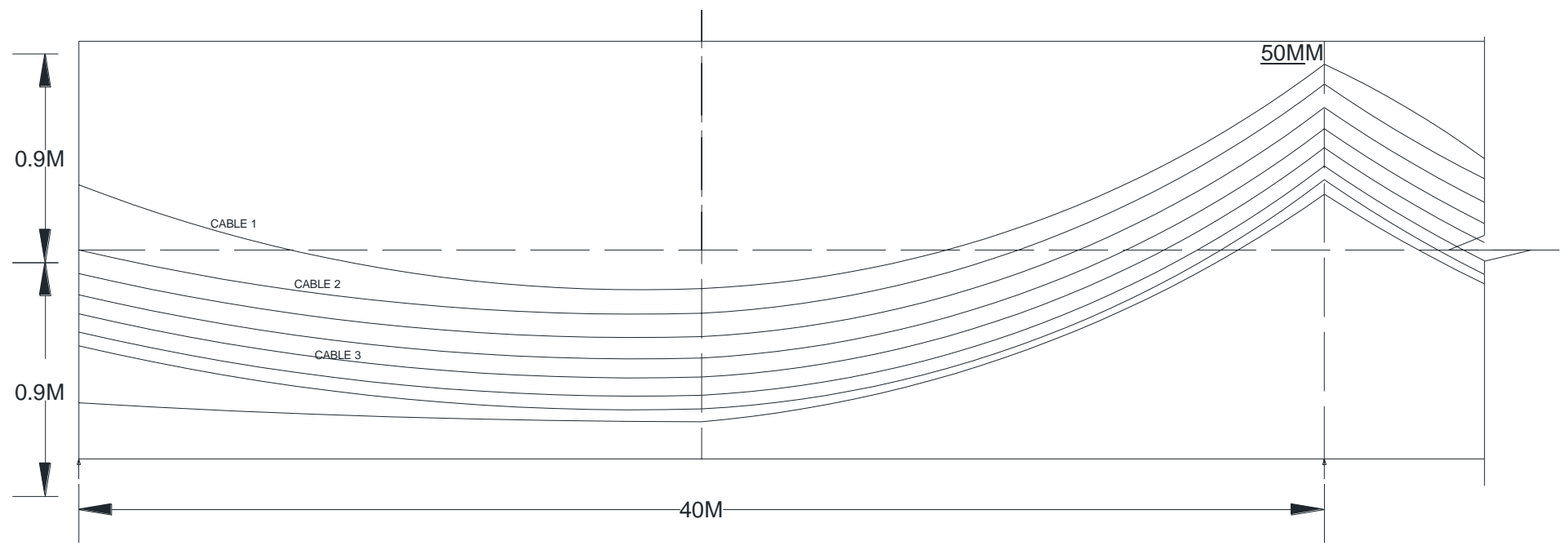

For the centre of span section,

\section{Check For Ultimate Flexural Strength:}

$\mathrm{Ap}=8 * 7 * 140=7840 \mathrm{~mm}^{2}$

$\mathrm{b}=1200 \mathrm{~mm} ; \quad \mathrm{d}=1600 \mathrm{~mm}$

$\mathrm{bw}=300 \mathrm{~mm} ; \quad \mathrm{fck}=50 \mathrm{~N} / \mathrm{mm}^{2} \mathrm{D}_{\mathrm{f}}=250 \mathrm{~mm}$

According to IRC: $18-2000, \mathrm{Mu}=1.5 \mathrm{M}_{\mathrm{G}}+2.5 \mathrm{M}_{\mathrm{L}}$

$=\left(1.5 * 8126 * 10^{6}\right)+\left(2.5 * 2809.04 * 10^{6}\right)$

$=19211.60^{*} 10^{6}$

$\equiv 19211.60 \mathrm{kN}-\mathrm{m}$

The ultimate flexural strength is computed as,

i) Failure by yielding of steel $\mathrm{Mu}=0.9 * \mathrm{~d}^{*} \mathrm{~A}_{\mathrm{p}} * \mathrm{f}_{\mathrm{p}}$

$=0.9 * 1600 * 7840 * 1862$

$=21021 \mathrm{kN}-\mathrm{m}$

ii)

Failure by crushing ofmaterials

$\mathrm{Mu}=0.176 \mathrm{~b}_{\mathrm{w}} \mathrm{d}^{2} \mathrm{f}_{\mathrm{ck}}+0.667 * 0.8 *\left(\mathrm{~b}-\mathrm{b}_{\mathrm{w}}\right)\left(\mathrm{d}-\mathrm{D}_{\mathrm{f}} / 2\right) \mathrm{D}_{\mathrm{f}} * \mathrm{f}_{\mathrm{ck}}$

$=(0.176 * 300 * 1600 * 50)+0.667 * 0.8 * 900 *(1600-(250 / 2)) *(250 * 250)$ 
$=\underline{51008 \mathrm{kN}-\mathrm{m}}$

According to IS: 1343-1980, the ultimate flexural strength of the centre span section is computed as,

$\mathrm{Ap}=\left(\mathrm{A}_{\mathrm{pw}}+\mathrm{A}_{\mathrm{pf}}\right)$

$A_{p f}=0.45 f_{c k}\left(b-b_{w}\right)\left(D_{f} / f_{p}\right)$

$=0.45 * 50 *(1200-300) *(250 / 1862)$

$=2718.85 \mathrm{~mm}^{2}$

$\therefore$ Apw $=7840-2718.85$

$=5121.15 \mathrm{~mm}^{2}$

Ratio, $\frac{A p w \cdot f p}{b w \cdot d \cdot f k}=\frac{5121.85 * 1862}{300 * 1600 * 50}$

$\underline{\underline{=0.397}}$

From table 11 of IS: 1343 , we have for post tensioned beams with effective bond, $\mathrm{fpu} / 0.87 \mathrm{fp}=0.9$ $\mathrm{fpu}=0.87 * 0.9 * 1862$

$=1457.95$

$\mathrm{X}_{\mathrm{u}} / \mathrm{d}=0.653$

$\mathrm{X}_{\mathrm{u}}=0.653 * 1600=$

$=1044.8 \mathrm{~mm}$

$\therefore \mathrm{Mu}=$ fpu. Apw. $\left(\mathrm{d}-0.42 \mathrm{X}_{\mathrm{u}}\right)+0.45 \mathrm{fck}(\mathrm{b}-\mathrm{bw}) \mathrm{Df}(\mathrm{d}-0.5 \mathrm{Df})$

$=[1457.95 * 5121.15 *(1600-0.42 * 1044.8)]+[0.45 * 50 * 900 * 250 *(1600-0.5 * 250)$

$=16134 * 10^{6} \mathrm{kN}-\mathrm{m}$

\section{Check For Ultimate Shear Strength:}

Ultimate Shear force $\mathrm{Vu}=(1.5 \mathrm{Vg}+2.5 \mathrm{Vq})$

$=(1.5 * 805.1+2.5 * 433.95)$

$\equiv 2292.525 \mathrm{kN}$ 
According to IRC: 18-2000, the ultimate shear resistance of support section uncracked in flexure is given by, $V c w=0.67 b w \cdot h \sqrt{ }\left(f_{t}^{2}+0.8 f c p . f_{t}\right)+\eta \cdot P \cdot S i n \Theta$ Where $b w=$ Width of web $=300 \mathrm{~mm}$

$\mathrm{h}=$ Overall depth of Girder $=1800 \mathrm{~mm}$

$\mathrm{f}_{\mathrm{t}}=$ Maximum principal tensile stress at centroid axis $\mathrm{f}_{\mathrm{t}}=0.24 \sqrt{ } \mathrm{f}_{\mathrm{ck}}=0.24 \sqrt{50}=1.7 \mathrm{~N} / \mathrm{mm}^{2}$

fcp $=$ Compressive stress at centroidal axis due toprestress

$=\frac{\mathrm{n}^{\mathrm{P}}}{A}$

$=\left(0.8 * 10826.94 * 10^{3}\right) /\left(0.845^{*} 10^{6}\right)$

$=10.25 \mathrm{~N} / \mathrm{mm}^{2}$

Eccentricity of cables atcentre of span $=850 \mathrm{~mm}$ Eccentricity of cables at support $=180 \mathrm{~mm}$

Net eccentricity $=\mathrm{e}=(850-180)=670 \mathrm{~mm}$ Slope of the cable $=\Theta=\frac{4 e}{L}$

$=\frac{48670}{40 \times 1000}$

$=0.067$

$\therefore \mathrm{Vcw}=0.67 * 300 * 1800 \sqrt{ }\left(1.7^{2}+0.8 * 10.25 * 1.7\right)+0.8 * 10826.94 * 10^{3} * 0.067$

$=2064.58 \mathrm{kN}$

Shear resistance required $=2292.525 \mathrm{kN}$ Shear capacity of section $=2064.58 \mathrm{kN}$

Base shear $=(2292.525-2064.58)=227.945 \mathrm{kN}$

Using 10mm diameter 2 legged stirrups of Fe415 HYSD bars, the spacing Sv is obtained as,

$\mathrm{Sv}=\left(0.87 * \mathrm{f}_{\mathrm{y}} * \mathrm{~A}_{\mathrm{sv}} * \mathrm{~d}_{\mathrm{t}}\right) / \mathrm{V}$

$=(0.87 * 415 * 2 * 79 * 1750) /\left(227.945 * 10^{3}\right)$

$=437.95 \mathrm{~mm}$

Provide $10 \mathrm{~mm}$ diameter stirrups at $150 \mathrm{~mm}$ centres near support and gradually increased to $300 \mathrm{~mm}$ towards the centre of span.

Provide $10 \mathrm{~mm}$ diameter stirrups at $150 \mathrm{~mm}$ centers near support and gradually increased to $300 \mathrm{~mm}$ towards the center of span. 


\section{DESIGN OF END BLOCKS:}

Solid end blocks are provided at the end supports over a length of $1.5 \mathrm{~m}$. Typical equivalent prisms on which the anchorage forces are considered to be effective are detailed in the figure. The bursting tension is computed using the data given in the table.

\begin{tabular}{|l|l|l|l|l|l|}
\hline$\left(\mathrm{y}_{\mathrm{po}} / \mathrm{y}_{\mathrm{o}}\right)$ & 0.3 & 0.4 & 0.5 & 0.6 & 0.7 \\
\hline$\left(\mathrm{F}_{\mathrm{bst}} / \mathrm{P}_{\mathrm{k}}\right)$ & 0.23 & 0.20 & 0.17 & 0.14 & 0.11 \\
\hline
\end{tabular}

In the horizontal plane we have the data, $\mathrm{P}_{\mathrm{k}}=1459 \mathrm{kN}$

$2 \mathrm{y}_{\mathrm{po}}=225 \mathrm{~mm} 2 \mathrm{y}_{\mathrm{o}}=900 \mathrm{~mm}$

$\therefore$ The ratio $\left(\mathrm{y}_{\mathrm{po}} / \mathrm{y}_{\mathrm{o}}\right)=(112.5 / 450)=0.25$ Bursting Tension, $\mathrm{F}_{\mathrm{bst}}=(0.23 * 1459)=336 \mathrm{kN}$

Area of stel required to resist this tension is obtained as, As $=\left(336^{*} 10^{3}\right) /(0.87 * 415)=931 \mathrm{~mm}^{2}$

Provide $10 \mathrm{~mm}$ diameter bars at $100 \mathrm{~mm}$ centres in the horizontal direction. In the vertical plane, the ratio of $\left(\mathrm{y}_{\mathrm{po}} / \mathrm{y}_{\mathrm{o}}\right)$ being higher the magnitude of bursting tension is smaller. However the same reinforcements are provided in the form of a mesh both in the horizontal and vertical directions.

\section{Cross Girders:}

The cross girder of width $200 \mathrm{~mm}$ and depth $1250 \mathrm{~mm}$ is provided with a nominal reinforcement of 0.15 percent of cross section consisting of $12 \mathrm{~mm}$ diameter bars spaced two at top, two at mid depth and two at bottom. Also provide nominal stirrup made up of $10 \mathrm{~mm}$ diameter two legged links at $200 \mathrm{~mm}$ centres. Two cables consisting of 12 numbers of $7 \mathrm{~mm}$ high tensile wires are positioned at mid third points along the depth. The transverse cables are straight along the length of cross girder.

\section{Design of Elastomeric Bearing:}

Selection of Bearing Pad dimension:

Maximum vertical load on bearing $=\mathrm{Nmax}=1240 \mathrm{kN}$

Select plan dimension of bearing pad of size $(400 \times 630) \mathrm{mm}$ Loaded area, $\mathrm{A}_{2}=23.9 * 10^{4} \mathrm{~mm}^{2}$

According to clause 307.1 of IRC: 21 , Allowable contact pressure $=0.25 \mathrm{fc} \sqrt{ }\left(\mathrm{A}_{1} / \mathrm{A}_{2}\right)$ Where $\mathrm{A}_{1}=$ Concrete bed block area over peir

$\mathrm{A}_{2}=$ Elastomeric pad area The ratio $\left(\mathrm{A}_{1} / \mathrm{A}_{2}\right)$ is limited to 2

Allowable contact pressure $=\sigma_{\mathrm{c}}=(0.25 * 20 \sqrt{ } 2)$

$=7.07 \mathrm{~N} / \mathrm{mm}^{2}$ Effective bearing area required $=\underline{\underline{\mathrm{Nax}} / \sigma_{\mathcal{C}}}$

$=\left(1240 * 10^{3}\right) / 7.07$ 
$=17.5^{*} 10^{4} \mathrm{~mm}^{2}<23.9 * 10^{4}$

Hence safe.

Bearing stress $=\alpha_{\mathrm{m}}=\left[\left(1240 * 10^{3}\right) /\left(23.9 * 10^{4}\right)\right.$

$=5.18 \mathrm{~N} / \mathrm{mm}^{2}$

According to IRC:83 clause 916.2, thickness of individual elastomer layers $h_{i}=12 \mathrm{~mm}$ Thickness of outer layer $=h_{\mathrm{e}}=6 \mathrm{~mm}$

Thickness of steel laminates $=\mathrm{h}_{\mathrm{s}}=3 \mathrm{~mm}$ Side covering $=\mathrm{c}=6 \mathrm{~mm}$

Adopt 3 laminates with two internal layers, Total thickness of elastomeric pad,

$H_{o}=\left(2 h_{e}+3 h_{s}+2 h_{i}\right)$

$=[(2 * 6)+(3 * 3)+(2 * 12)]$

$=45 \mathrm{~mm}$

Shape factor $=\frac{\text { loaded surface area of an internal layer of elastomer }}{\text { Surface area free to bulge }}$

$=\left[\frac{(630-12)(400-12)}{(2 * 12)(630 * 400)}\right]$

$=9.7>6<12$

Hence safe.

Shear strain due to creep, shrinkage and temperature per bearing,

$=\left(0.5 * 6 * 10^{-4}\right)$

$=3 * 10^{-4}$

Shear strain due to translation per bearing $\gamma_{\mathrm{d}}=($ Shear strain due to creep shrinkage and temperature $)+($ Shear strain due to longitudinal force)

$\left.=\left[\frac{(0.0003)(16000)}{(45)}\right]+\frac{(45000)}{(239000)}\right]$

$=0.106+0.188$

$=0.294 \mathrm{~N} / \mathrm{mm}^{2}$

Assuming $\sigma_{\max }=10 \mathrm{~N} / \mathrm{mm}^{2}$

Maximum permissible angle of rotation of a single internal layer of elastomeric Corresponding to $\sigma_{\mathrm{m}}$ value 
Of $10 \mathrm{~N} / \mathrm{mm}^{2}$ is given by

$\alpha_{\text {bi.max }}=\left[\frac{0.5 \sigma m h i}{(b . s . s)}\right]$

$=(0.5 * 10 * 12) /\left(388 * 9.7^{2}\right)$

$=0.001643$ radians Permissible rotation $=\alpha_{d}=\beta . \eta_{.} \alpha_{\text {bi.max }}$ Where $\beta=0.1 \sigma_{\mathrm{m}}$

$=0.1 * 5.18$

$=0.518 \mathrm{~N} / \mathrm{mm}^{2}$

And $\mathrm{n}=$ number of internal elastomeric layers $=2 \alpha_{\mathrm{d}}=(0.518 * 2 * 0.00164)$

$=0.00169>0.0015$ (Actual). . . Hence safe.

\section{Friction:}

Shear strain computed $=0.294$ Under critical loading conditions, Shear strain $<=0.2+0.1 \sigma_{\mathrm{m}}$

$<=0.2+0.1 * 5.18$

$<=0.718>0.294$

Hence safe.

Also $\sigma_{\mathrm{m}}=5.18 \mathrm{~N} / \mathrm{mm}$, satisfies the criteria that $10 \mathrm{~N} / \mathrm{mm}^{2}>=\sigma_{\mathrm{m}}>=1.5 \mathrm{~N} / \mathrm{mm}^{2}$ Total shear stress,

Shear stress due to compression $=1.5\left(\sigma_{\mathrm{m}} / \mathrm{S}\right)$

$=1.5(5.18 / 9.70)$

$=0.80 \mathrm{~N} / \mathrm{mm}^{2}$ Shear stress due to horizontal deformation,

$=\tau_{\mathrm{r}}=\gamma_{\mathrm{d}}=0.294 \mathrm{~N} / \mathrm{mm}^{2}$ as per computation due to translation. Shear stress due to rotation $=0.5^{*}(\mathrm{~b} / \mathrm{hi})^{2 *} \alpha_{\mathrm{bi}}$

$=0.5(388 / 12)^{2} * 0.00164$

$=0.857 \mathrm{~N} / \mathrm{mm}^{2}$

Total shear stress $=(0.80+0.294+0.857)$

$=1.95 \mathrm{~N} / \mathrm{mm}^{2}<6 \mathrm{~N} / \mathrm{mm}^{2}$

Hence adopt an elastomeric pad bearing of overall dimensions (400 x 630)mm with a total thickness of $45 \mathrm{~mm}$ having two internal elastomeric layers of $12 \mathrm{~mm}$ thickness and three steel laminates of thickness $3 \mathrm{~mm}$ each having bottom and top cover of $5 \mathrm{~mm}$. 


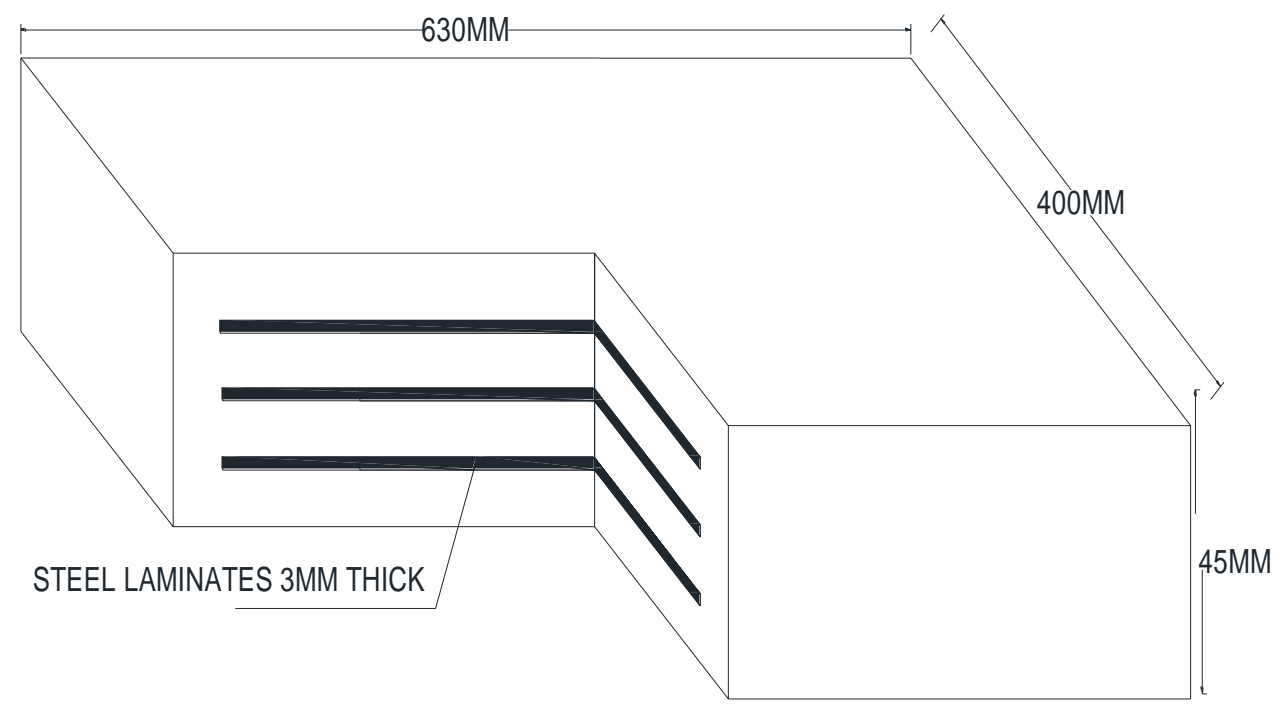

ELASTOMERIC PAD BEARING

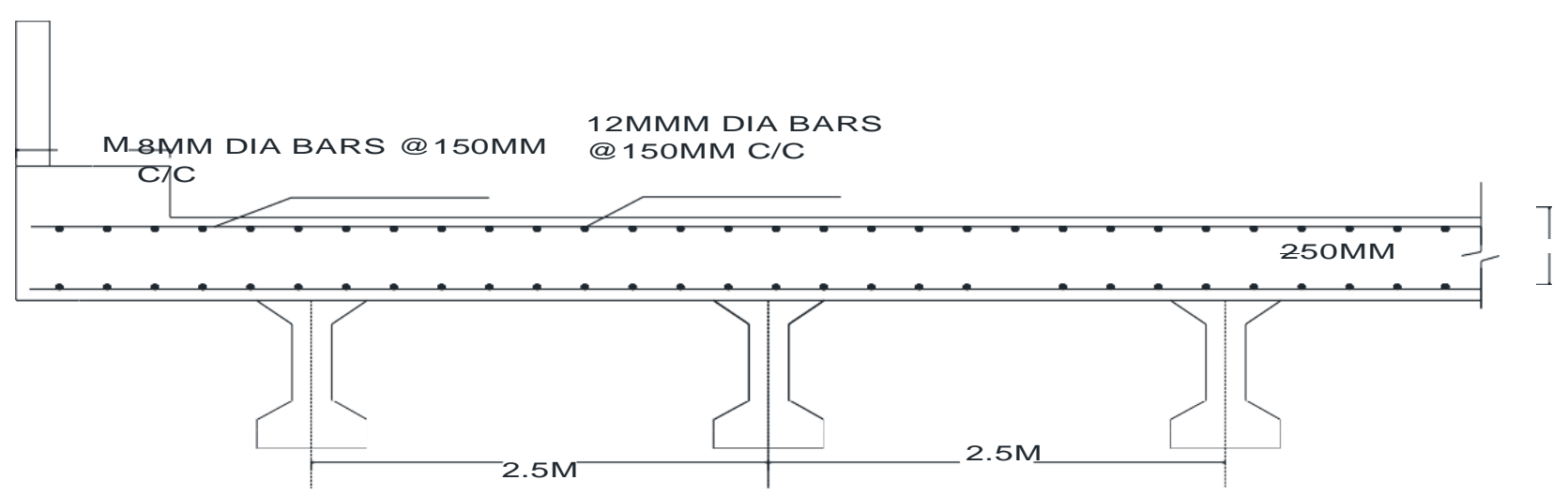

\section{CROSS-SECTION OF DECK \\ SLAB}




\section{CONCLUDING}

In this project efforts have been made to analyse and design the PSC-1 beam-bridgetaking into consideration IRC Class AA tracked and wheeled vehicle loads. The analysis and design is done for worst combination of loads. The proposed bridge is a two-lane bridge which is sufficient enough to accommodate present traffic conditions.

\section{REFERENCES}

[1]. Design of prestressed concrete structure- t.y.lin and nedh.burns.

[2]. Design of prestressed concrete structure -krishnaraju.n.

[3]. Design of bridges -N. Krishna raju.

[4]. Essentials of bridge engineering- D.Johnsonvictor.

[5]. IS 456-2000, plain reinforced concrete- code forpractice.

[6]. IRC 6-2000 standard specifications and code of practice for road bridges-loads andstresses.

[7]. IRC 21-2000 standard specifications and code of practice for road bridges- cement concrete (plain and reinforced).

[8]. IRC 78-2000 standard specifications and code of practice for road bridges- foundations andsubstructure.

[9]. IRC 18-2000 design criteria for prestressed concrete bridges (post tensioned member).

[10]. IS 1343-1980 indian standard code of practice for pre stressesconcrete.

[11]. IS 1785-1983 indian standard specifications for plain and hard drawn steel wire. 